
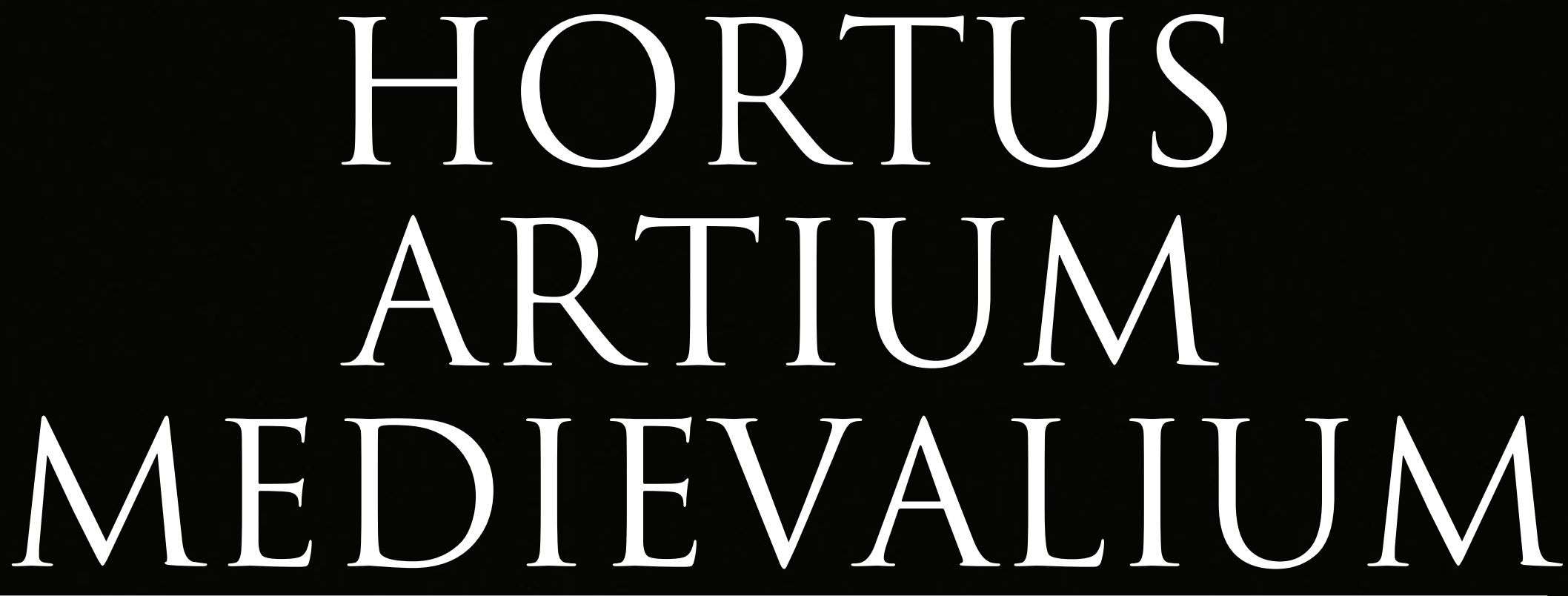

Journal of the International Research Center for Late Antiquity and Middle Ages, Vol. 20/2, Zagreb-Motovun, 2014. 


\section{HORTUS ARTIUM MEDIEVALIUM}

Journal of the International Research Center for Late Antiquity and Middle Ages 
Journal of the International Research Center for Late Antiquity and Middle Ages

Volume: 20/2, 2014

Publisher:

International Research Center for Late Antiquity and Middle Ages, Motovun;

University of Zagreb, Croatia

Editor:

Miljenko Jurković,

Department of Art History, Faculty of Humanities and Social Sciences, University of Zagreb, Croatia

Editorial Board:

Jean-Pierre Caillet (Paris)

Igor Fisković (Zagreb)

Nikola Jakšić (Zadar)

Miljenko Jurković (Zagreb)

Giovanna Valenzano (Padova)

Fulvio Zuliani (Padova)

Council of the International Center for Late Antiquity and Middle Ages:

Xavier Barral i Altet, professeur émérite d'Histoire de l'art du Moyen Age à l'Université de Rennes 2, Visiting professor Università di Venezia Ca'Foscari

Gian Pietro Brogiolo, professore di archeologia medievale, Università degli Studi di Padova, Italia

Noël Duval, professeur émérite d'archéologie de l'Antiquité tardive à l'Université de Paris IV-Sorbonne, France

Alain Erlande-Brandenburg, directeur des études, Institut d'Etudes Supérieures des Arts, Paris, France

Igor Fisković, member of the Croatian Academy of Sciences and Arts, professor of Medieval Art History, Faculty of Humanities and Social Sciences, University of Zagreb, Croatia

Nikola Jakšić, professor emeritus of Medieval Art History, University of Zadar, Croatia

Miljenko Jurković, professor of Medieval Art History, Faculty of Humanities and Social Sciences, University of Zagreb, Croatia, President of the Council

Gisela Ripoll-López, profesora titular de arqueología, Universitat de Barcelona, España

Fulvio Zuliani, professore di storia dell'arte medievale, Università degli Studi di Padova, Italia

Financial Support:

Ministry of Science, Education and Sports of the Republic of Croatia

Language Consultants: Pascale Chevalier (French), Jan Vanek (Italian), Vesna Mildner (English)

\author{
Editorial assistant: Maja Zeman \\ Computer layout: Boris Bui \\ Printing: Kerschoffset, Zagreb-Lučko, Croatia
}

Issued: May 2014, 600 copies

Correspondence and Exchanges:

Faculty of Humanities and Social Sciences, University of Zagreb, Department of Art History, (Dr. Miljenko Jurković), Ivana Lučića 3, 10000 Zagreb, Croatia, Phone: ++385 16120 147, Fax: ++385 16120144.

\title{
Adresse de diffusion:
}

Adresser toute correspondance commerciale (commandes, abonnements) aux Éditions Brepols Begijnhof 67, B-2300 Turnhout (Belgique), e-mail : periodicals@brepols.net

Hortus Artium Medievalium is published annually. Articles are reviewed internationally.

Cover: Rome, Arch of Constantine, north side, Vicennalia

HAM is indexed in:

BHA, Bibliography of the History of Art - Bibliographie d'Histoire de l'Art, Vandoeuvre-lès Nancy Cedex, France, Santa Monica, Cal. USA Architecture Database. Architectural Publications Index \& Architectural Publications Index on disc (CD/ROM), Royal Institute of British Architects, The British Architectural Library.

Art and Archaeological Technical Abstracts (“AATA”). Getty Conservation Institute Projects Bibliographies ERIH - European Reference Index for the Humanities

FRANCIS database (INIST, CNRS) 


\section{CONTENTS}

\section{THE (R)EVOLUTION IN CHRISTIAN RELIGIOUS ARCHITECTURE AND LITURGY}

M. Jurković, The (R)evolution in Christian Religious Architecture and Liturgy - Introduction

W. Jacobsen, L'arredo ecclesiatico nello sviluppo della liturgia tra tarda Antichità e Alto Medioevo annotazioni di metodo

N. Reveyron, Forma monasterii. Essai sur l'organisation de l'espace monastique comme mise en forme de l'identité ecclésiologique Review

G. Archetti, Spazi e strutture claustrali nei commenti carolingi alla Regola benedettina Original scientific paper

I. Marić, S. Bully, M. Jurković et M. Čaušević-Bully, le monastère saint-Pierre d'Osor (île de Cres) : huitième campagne d'études archéologiques Preliminary communication

S. Bully, Un dispositif funéraire spécifique : les formae. État de la question et nouvelles découvertes Preliminary communication

C. Tosco, The Cross-in-square Plan in Carolingian Architecture Preliminary communication

C. Ebanista, la basilica sub divo nel complesso cimiteriale di S. Gennaro a Napoli: spazio liturgico, culto martiriale e utilizzo funerario Original scientific paper

F. Chantinne, Ph. Mignot, La collégiale Sainte-Gertrude de Nivelles. Réexamen du dossier archéologique Preliminary communication

Ph. George, De constructione - de consecratione ecclesiae Hoyensis (1066) Original scientific paper

T. Creissen, E. Lorans, L'apport des dernières fouilles archéologiques à la connaissance des églises abbatiales de Marmoutier antérieures à la reconstruction gothique Preliminary communication

A. Falchi, Montecassino e l'architettura campana di XI secolo: il caso di San Benedetto a Salerno Preliminary communication

G. Mallet, Héritages haut-médiévaux dans les édifices romans de Catalogne du Nord. Le cas de l'église abbatiale bénédictine à double chevet Sainte-Marie d'Arles-sur-Tech Preliminary communication

Y. Kojima, Insediamenti monastici nell' Emilia occidentale - il monastero benedettino di Castione Marchesi e i cistercensi Preliminary communication

S. Demarthe, Réflexions autour des églises dites « à passages » : le cas de Saint-Pierre de Corgoloin (Côte-d'Or) Preliminary communication

P. Marković, K. Karlo, Religious Architecture of the military Orders in Medieval Slavonia and its Reflections in the $13^{\text {th }}$ and $14^{\text {th }}$ Century Preliminary communication

N. Jakšić, A Ninth-century Stone-cutting Workshop in Southern Dalmatia Original scientific paper

P. Vedovetto, Elementi di arredo liturgico altomedievale e preromanico dalla chiesa di San Martino a Padova: rilettura complessiva dei materiali

Preliminary communication

R. Cassanelli, Architettura e scultura romanica nel Contado del Seprio. Nuove considerazioni sul complemento plastico della chiesa monastica di Cairate Original scientific paper

M. Vaccaro, "The most charming Romanesque mosaic": iconografia e restituzione grafica del mosaico pavimentale di San Tommaso ad Acquanegra sul Chiese. Preliminary communication

I. Lorés Otzet, From Roda de Ribagorça to Lleida (XII-XIII Centuries): Artistic and Liturgical Transfers between Displaced Episcopal Sees Original scientific paper

IMAGES OF CHRISTIANITY AND THE (RE) MAKING OF CHRISTIAN IDENTITY

J.-P. Caillet, Images of Christianity and the (re)making of Christian identity 
B. Brenk, Early Christian Mosaics: A Floating World of Abstract Associations Original scientific paper

Y. Christe, Le grand portail de Cluny III : les interférences de la tradition tardo-antique, romaine et islamique sur un monument atypique

Original scientific paper

A.-O. Poilpré, Que disent les images bibliques de l'identité chrétienne, aux III et IVe siècles ? Preliminary communication

J. Leclercq-Marx, L'intégration des Sept Merveilles du Monde à la culture chrétienne. Entre survivance et réinterprétation Original scientific paper

L. Bartolomé Roviras, The Works of the Master of the Tympanum of Cabestany. A Mirror of the Roman Sarcophagi sculpture Preliminary communication

F. Stroppa, Aspetti del mondo rurale nelle fonti artistiche tra storia e storiografia (secoli IX-XV) Review

M. Gianandrea, Nel lusso della tradizione. L'inedita decorazione del nartece di Santa Sabina all'Aventino a Roma (Il nartece di Santa Sabina, I) Original scientific paper

I. Foletti, Le porte lignee di Santa Sabina all'Aventino: tra liturgia stazionaria e funzione iniziatica (Il nartece di Santa Sabina, II) Preliminary communication

G. P. Brogiolo, V. Gheroldi, F. De Rubeis, J. Mitchell, Nuove ricerche su sequenza, cronologia e contesto degli affreschi di Santa Maria foris portas di Castelseprio Original scientific paper

M. Angheben, La théophanie absidale de Galliano. Les archanges-avocats transmettant les prières du Pater et l'Église céleste célébrant le sacrifice eucharistique Original scientific paper

S. D'Ovidio, Spazio liturgico e rappresentazione del sacro : crocifissi monumentali d'età romanica a Napoli e in Campania Preliminary communication

S. Piazza, L’Albero di Iesse nel XII secolo fra Occidente e Oriente: note sul perduto mosaico della basilica della Natività a Betlemme Original scientific paper

V. Lucherini, Il polittico portatile detto di Roberto d'Angiò nella Moravská galerie di Brno: questioni di araldica, committenza e iconografia Original scientific paper

J. Molina Figueras, Iconos marianos, leyendas y monarquía en la Corona de Aragón (s. XIII-XV) Preliminary communication

D. Allios, De la nation à la région, l'Histoire de l'art, archéologie et mythes fondateurs Review

VENICE AND THE ADRIATIC IN THE MIDDLE AGES

S. Riccioni, Venezia e l'Adriatico nel Medioevo

D. Calaon, Ecologia della Venetia prima di Venezia: uomini, acqua e archeologia Original scientific paper

M. Agazzi, Torcello medioevale, scultura e architettura Original scientific paper

F. Coden, Dall'Oriente all'Occidente: capitelli ad incrostazione di mastice a nord di Venezia Original scientific paper

D. Valenti, I motivi decorativi del duecento nei mosaici di San Marco a Venezia Original scientific paper

L. V. Geymonat, Il primo bagno di Gesù a Traù e Venezia Original scientific paper

L. Palozzi, Venetian or Adriatic? Refocusing the Geography of Late-Medieval Stone Sculpture in the Adriatic Basin: Four Case Studies Preliminary communication

Z. Murat, The Tomb of the Beato Pacifico in the Basilica dei Frari: Personal Devotion or Public Propaganda? Original scientific paper 


\section{ECOLOGIA DELLA VENETIA PRIMA DI VENEZIA: UOMINI, ACQUA E ARCHEOLOGIA}

\section{DIEGO CALAON}

UDC: 711.52:902(450.341)"02/09" $728.1(1-21)(450.341)^{\prime \prime} 02 / 09^{\prime \prime}$ Original scientific paper

Manuscript received: 03. 04. 2014.

Revised manuscript accepted: 28. 04. 2014.

DOI: 10.1484/J.HAM.5.102695
D. Calaon

DAIS - Department of Environmental Studies, Ca' Foscari Venezia Dorsoduro 2137, 30123 - Venezia, Italia Department of Anthropology, Stanford 450 Serra Mall, Stanford, CA 94305, USA

This paper explores a new perspective on the problem of the origins of the Venetian settlements before Venice's birth. With an archaeological viewpoint, the author considers the recent excavation data from Torcello (2012-2013 campaigns), integrating modern debates on ecological degradation and anthropological theory of materiality. The attempt is to reconsider the process of Europeanization through the formation of new settlements in the Venetian lagoons at the end of the Roman period.

The paper focuses on the relationship between the environment and the societal structures from Late Antiquity to the Early Middle ages. During this critical period of flux, it is possible to study how ethnic and social groups developed a new social geography and how new political systems and different trade networks shaped the "rising Venice".

Keywords: Venice, Torcello, Late Antiquity and Early Middle Age settlements, Venice's origins, Landscape and Ecology

\section{UN PROBLEMA ASSAI COMPLESSO ${ }^{1}$}

Il nesso storico tra Venezia el'acqua è più che evidente. Il legame tra ambiente marino, laguna e città è stato al centro di innumerevoli studi sulla Serenissima. Analisi storiche e archeologiche, recenti e passate, hanno sottolineato come la collocazione topografica all'interno della laguna abbia significato per la città molto più di una fortuita sede ${ }^{2}$. L'acqua e il mare sono gli elementi costitutivi dei processi economici veneziani. Nell'evoluzione del complesso rapporto tra gli uomini e l'ambiente lagunare è possibile rintracciare le condizioni che definiscono la straordinaria parabola di Venezia.

Se, però, spostiamo la nostra attenzione agli studi che si sono occupati del problema delle origini delle città lagunari, la complessità del rapporto uomo/acqua tende a ridursi - soprattutto nelle ricostruzioni degli storici di lingua italiana - a una dicotomia piuttosto semplificata, che oppone la terraferma alla laguna. Da tale opposizione discende una sorta di contrapposizione tra due categorie di "fenomeni storici".

Nel tradizionale racconto delle origini di Venezia, da una parte, nell'entroterra, in età romano-imperiale si sarebbero trovate le ricche città romane, i commerci, le comunicazioni, la fertile campagna, gli insediamenti agricoli, e gli spazi agrari centuriati. Nei secoli successivi - sempre nell'entroterra - si sarebbero incontrati i funesti segni delle invasioni germaniche con distruzioni, abbandoni delle vie di comunicazione e delle campagne, de-popolamento delle città e, infine, un generale imbarbarimento della "natura".

Dalla parte della laguna, invece, si sarebbero contrapposti isolotti sicuri, circondati da acque salse e sufficientemente remoti da costituire una difesa naturale. Le isole lagunari, sarebbero state percepite come poco abitate nell'antichità e, di norma, immaginate come colonizzate da una popolazione di "pionieri" capaci di "adattarsi" alle nuove e dure condizioni ambientali. Nei secoli dell'alto medioevo, infine, tali isolotti sarebbero stati nobilitati dalla presenza di chiese e vescovi, citati dalla storiografia locale come simboli di una rinnovata civiltà ricostruita, basata su principî quali la libertà e l'indipendenza.

Tale percezione della laguna, però, non è un'esclusiva degli storici. Immaginare la laguna antica come luogo inospitale, ma sicuro è un'opinione difusa. Le isole tra le acque salse avrebbero costretto a precarie abitazioni in legno e a una continua lotta contro la marea per strappare pochi metri di terra calpestabile. Si tratta di un'idea condivisa da tutti i veneziani da molte generazioni.

Il mito di fondazione della città vuole che Venezia sia nata dall'azione dei profughi che qui si sarebbero rifugiati per scampare alle orde barbariche: lo si impara sui banchi di scuola alle elementari, lo si apprende dai racconti orali, lo si legge oggi in tutti i siti internet, a partire dalle pagine di Wikipedia fino ai numerosissimi siti turistici ${ }^{3}$. Attila, Alboino, Agilulfo a capo di Unni e di Longobardi sarebbero coloro che avrebbero costretto alla difesa nelle lagune: le isole sarebbero diventate "castelli" difesi dalle acque. Anche le sintesi storiche più aggiornate, capaci di distinguere da ciò che fa parte del bagaglio del mito delle origini, incrostatosi ormai tra le pieghe della storia istituzionale, $\mathrm{e}$ i dati desumibili dai pochi documenti scritti conservatisi nell'alto medioevo, non riescono a "non" considerare la laguna come un luogo "altro", "segregato e sicuro"4 rispetto alla terraferma.

E' ovvio, dunque, il peso del mito nei processi di ricostruzione storica. Va detto, però, che il mito della fondazione della città, elaborato in seno alle cancellerie veneziane a partire dall'XI secolo, non fa esplicitamente riferimento a un ambiente lagunare "ostile" all'insediamento. Giovanni Diacono, che esalta le origini dei Veneziani, ci elenca insulae, civitas, castra, flotte, rotte marine e lagunari: non pare interessato a sottolineare un'eventuale difficoltà nell'abitare le lagune ${ }^{5}$. Una certa precarietà degli abitati lagunari (ma sarebbe meglio dire "peculiarità"), si percepisce, piuttosto, da altre fonti più antiche di area bizantina. Cassiodoro, ad esempio, è quasi affascinato dall'integrazione delle acque salse nella vita quotidiana, nelle scelte economiche e nei saperi tecno-costruttivi dei Venetici che popolano le lagune 
tra Aquileia e Ravenna ${ }^{6}$. Possiamo sostenere con una certa sicurezza, invece, che si debba alla rilettura del mito di fondazione in chiave marcatamente isolazionistica, prodotta nel XX secolo, la consacrazione definitiva della laguna come spazio "di rifugio", spazio separato dove custodire le tradizioni bizantine, spazio contrapposto alla barbarie del nuovo entroterra Longobardo? .

\section{CITTÀ (EMPORI) SULL'ACQUA: LE ROTTE BIZANTINE DELLA TRADIZIONE STORIOGRAFICA}

Le acque della laguna, dunque, nella storiografia delle origini sono percepite come un luogo necessario ma svantaggioso: pur di mantenere la libertà e i legami con un oriente bizantino (fonte di ricchezze e cultura) i rifugiati si sarebbero improvvisati pescatori e salinatori. Pionieri che, sempre secondo una consolidata storiografia, ben presto si sarebbero liberati dell'immagine di "fuggiaschi nelle acque" per diventare "padroni delle acque", destinati a dominare il Mediterraneo. Ma anche in questo caso il mito (e i suoi riflessi politico-sociali di epoca contemporanea) tende a sovrastare la notizia storica: Venezia delle origini è davvero un "prodotto" bizantino? E, soprattutto, quali sono i caratteri di bizantinità degli spazi agrari e lagunari venetici tra VI e VIII secolo?

Sicuramente sono bizantine le titolarità di alcuni dei primi componenti delle élite aristocratiche che partecipano alla creazione delle magistrature venete. Lo sono anche certuni titoli onorifici orientali che ricorrono nelle Cronache, anche se la verifica puntuale del loro effettivo peso materiale è difficile. Sono titoli che si richiamano con forza alla corte di Bisanzio: tribunus, magister militum ma anche spathario o ipato. Lo stesso Orso, doge tra il 726/7 e il 737, è associato a quest'ultimo titolo. E' forse il primo doge di cui, al di la della leggenda, è possibile tracciare un profilo storico di rilievo. Orso è al comando di una imponente flotta militare, la stessa che sarà in grado di soccorrere Ravenna a metà dell'VIII secolo. Orso esercita sicuramente un ruolo di rilievo nell'esercito, oltre che un ruolo di capo politico e amministrativo. E' quasi impossibile, però, stabilire quale sia il suo legame effettivo (politico ed economico) con Bisanzio. La corte sul Bosforo da tempo pare essere poco interessata al destino dell'alto Adriatico.

Pochi anni dopo, quello stesso titolo di doge sarà portato dai membri della famiglia dei Partecipazi. A loro si deve la fondazione vera e propria di Venezia, tra 810 e 830. Il doge Agnello Partecipazio sposta il centro dei suoi interessi economici (privati? pubblici?) in un gruppo di isole intorno a Rivoalto. Il $d u x$ sceglie un luogo nel mezzo della laguna, lungo un canale di marea connesso all'antico Brenta-Medoacus, dove fa erigere - in un lasso di tempo veramente breve - un nuovo palazzo degno del potere che incarna. Accanto a tale palazzo il $d u x$ fa avviare i lavori che daranno corpo a una cappella palatina di straordinaria importanza, la futura basilica di San Marco. L'edificio religioso non sarà solo abbellito e adornato con opere d'arte della tradizione altomedievale adriatica (più di stile adriatico/carolingio che bizantino ${ }^{8}$ ), ma ben presto ospiterà addirittura il corpo di uno degli Evangelisti. Quel corpo sarebbe fatto trafugare da Alessandria d'Egitto con l'intervento di commercianti veneziani. Qualche anno dopo, ancora a Rivoalto, quello stesso dux patrocina la nascita della prima moneta coniata in laguna, un denaro franco che sul verso ricorda il luogo di emissione (Venecias) e sul diritto indica chiaramente l'autorità emittente, Ludovico il Pio re dei Franchi ${ }^{9}$. Da questo momento possiamo parlare di un emporio adriatico ormai istituzionalizzato e riconosciuto dalle massime autorità dell'epoca: non è dato di poca importanza il fatto che in quel luogo si batta una moneta carolingia. Va ricordato che la zecca di Ravenna ha coniato le ultime monete negli anni '50 del 700, e queste erano emissioni longobarde ${ }^{10}$.

Il doge Agnello Partecipazio riuscirà a trasmettere il titolo al figlio, Giustiniano. Giustiniano era stato nominato ipato (console/governatore) dallo stesso imperatore di Costantinopoli, Leone V. Giustiano, dunque, è stato un doge Bizantino? Carolingio? Longobardo? Venezia di IX secolo è davvero una città (o quasi città) che possiamo definire bizantina?

Cittanova (la mitica Eraclea) - centro in cui la tradizione vorrebbe collocare l'elezione del primo mitico doge Paulicio - è davvero un' enclave bizantina fieramente contrapposta alla barbarie del vicino Ducato trevigiano? Più semplicemente, si tratta di uno spazio agrario/lagunare piuttosto periferico che, nel passaggio tra V e VII secolo, partecipa - nella sua dimensione territoriale - al nuovo equilibrio tra vecchi poteri militari (bizantini) e nuove élite (longobarde), esprimendo materialmente un accentramento della popolazione intorno ad un nucleo di potere politico e amministrativo (e forse anche religioso).

Le mitiche origini bizantine di Cittanova (anch'essa sarebbe nata con un atto fondativo ad opera dei profughi bizantini di Opitergium in fuga dopo le devastazioni longobarde) contrastano in maniera evidente con i risultati archeologici delle numerose indagini condotte sul terreno. La città (o villaggio agrario) corrisponderebbe a un insediamento di lunga durata che ha origine dalla concentrazione, in un unico centro di potere, di funzioni agrarie e forse commerciali. Cittanova si sviluppa in un' area ricca di infrastrutture agricole e per lo sfruttamento delle acque lagunari, con una cronologia che parte almeno dal III sec. d.C. Tra VI e VII secolo d.C. appaiono i primi edifici religiosi, tra cui un battistero che segnala la presenza di una comunità stanziale ormai fortemente legata al territorio. Non ci è dato conoscere la composizione sociale delle élite che guidano l'abitato in età altomedievale, ma numerosi sono i segni che potrebbero farle identificare con dignitari militari di area bizantina e, quindi, con spazi territoriali di pertinenza del fisco imperiale. Il luogo, infine, non si è mai chiamato Eraclea: le penne degli storici medievali al servizio di Venezia hanno probabilmente creato questa nomenclatura che avrebbe nobilitato il luogo dove sarebbe nata l'istituzione dogale, la più alta delle istituzioni politiche veneziane ${ }^{11}$.

Ele acque in cui si muovono i veneziani "prima diVenezia", fino a che punto sono bizantine? Venezia è presentata dalla tradizione storica attraverso un legame indissolubile con Costantinopoli, che sarebbe divenuto il suo naturale (e quasi unico) punto di riferimento. Venezia parrebbe avere rivolto le spalle all'entroterra padano. La principale destinazione dei commerci della marineria cittadina, nel pieno medioevo è il Bosforo. Non sembra, però, che i primissimi viaggi dei veneziani fossero tutti diretti a Costantinopoli. Tali viaggi, infatti, già partire dall'VIII secolo e per tutto il IX, tendono a puntare soprattutto verso le coste arabe del Mediterraneo meridionale, con Alessandria d'Egitto e l'attuale Israele (all'epoca sotto l'influenza araba) quali porti principali di destinazione ${ }^{12}$.

\section{L'ACQUA DELLARCHEOLOGIA: UNA PROPENSIONE PADANA}

Alla luce dell'archeologia, però, tale impostazione - che vedrebbe nella laguna il luogo remoto della bizantinità altri- 
menti perduta, può essere riletta e riconsiderata. Le recenti e meno recenti indagini stratigrafiche sottolineano un processo di continuità nell'insediamento lagunare, secondo una tendenza già chiara a partire dagli scavi degli anni '60 del secolo scorso ${ }^{13}$, e oramai comunemente accettata ${ }^{14}$.

L'archeologia, inoltre, suggerisce una percezione delle lagune in maniera assai diversa da quanto tradizionalmente riportato dalle fonti storiche interpretate. Il popolamento della laguna si rivela come il frutto di una scelta economica, sul lungo periodo, che mira a sfruttare le risorse ambientali delle acque interne. Dunque, l'archeologia descrive siti che prosperano sull'acqua, non che sopravvivono "nonostante" l'acqua ${ }^{15}$.

La compagine sociale da cui trae origine il popolamento è sicuramente eterogenea $\mathrm{e}$, in massima parte, non corrisponde solo ai "liberi cittadini" in fuga, quanto piuttosto a masse di lavoratori de-localizzati da élite imprenditoriali ${ }^{16}$.

Lo spazio lagunare è tutt'altro che vuoto, sconosciuto e marginale: al contrario pare inserirsi in un sistema di proprietà demaniali tardo antiche che è oggetto di uno specifico interesse da parte delle aristocrazie locali e da parte dei poteri politici regionali e internazionali ${ }^{17}$.

Prima del IX secolo, la frammentarietà (e, dunque, le differenze interne) tra i diversi siti che popolano la laguna è un dato di fatto. Ciò che invece pare accomunare tutti gli insediamenti è una certa "distanza" da Bisanzio (in termini di economia reale) e, nel contempo, una fortissima compenetrazione con l'entroterra padano (e dunque con quel che resta delle sue città). I commerci e le rotte dei veneziani delle origini sembrano orientati non ad una risposta economica solo alla domanda delle élite bizantine adriatiche, ma da una necessità di "contatti" (e scambi commerciali) tra tale entroterra (e le aristocrazie che lo governano) con un Mediterraneo arabo e bizantino ${ }^{18}$.

In termini archeologici e topografici, l'acqua può essere considerata il legame con l'entroterra: le vie di transito endolagunari mettevano in comunicazione merci e uomini con i canali e i fiumi navigabili della pianura. Si tratta di un'acqua che, se sufficientemente profonda, garantiva la mobilità, i contatti e gli scambi. Non per nulla l'archeologia delle Venezie prima di Venezia è costituita essenzialmente da waterfront, moli, rive, scivoli d'alaggio, porti, canali artificiali, punti d'approdo ${ }^{19}$.

L'acqua, come emerge dai canali a contatto delle strutture scavate dagli archeologi è un indice di unione e non di divisione. Le vie d'acqua segnano luoghi aperti ai commerci, regolati da scali e infrastrutture portuali (statio, cavane, approdi). La laguna non sembra essere stata percepita come elemento di "difesa": sovente si è scritto di come i siti lagunari non avrebbero avuto bisogno di proteggersi con mura e allestimenti castrensi, poiché la loro insularità era un deterrente sufficiente contro possibili invasioni o saccheggi.

Torcello è sicuramente aperta sul lato del Canal Grande, verso le rotte di navigazione lungo il Sile. A Cittanova fino ad oggi non sono emerse tracce di mura urbane. Le mura di Venezia costituiscono una sorta di costruzione storiografica assai tarda, senza però alcun riscontro archeologico. Comacchio non presenta apprestamenti difensivi, se non quelli emersi presso la cattedrale e costruiti a partire dalla fine del X secolo, e probabilmente legati alla difesa del centro religioso dagli attacchi "interni" dalla laguna (sferrati da Venezia che mira al dominio dei commerci lagunari e padani ${ }^{20}$ ). Mura, invece, sono presenti a Grado: i dati raccolti relativi alla cinta muraria della città indicano che sarebbe stata costruita in un' unica soluzione, databile alla seconda metà del VI sec., ovvero in un momento in cui molte altre città costiere romane si dotano di apprestamenti difensivi. Grado è in realtà un centro tardo romano, costituisce il porto satellite di Aquileia già a partire dal IV secolo d.C. ${ }^{21}$.

I centri della Venetia post-classica, sia quelli che si formano già a partire dalla tarda antichità (Cittanova, Jesolo, Torcello, San Basilio, Santa Maria in Padovetere), sia quelli che nascono in piena età altomedievale, sorgono lungo le vie di comunicazione più frequentate, all'imbocco di fiumi navigabili, in luoghi aperti alla viabilità dell'entroterra padano. Non è un caso che una delle fondazioni più importanti legate al doge Agnello Partecipazio, il monastero dei SS. Ilario e Benedetto (fineVIII-inzio IX secolo), sito dove lo stesso doge e alcuni dei sui discendenti saranno sepolti, si trovi in un'isola presso i rami minori dell'antico MedoacusBrenta, sulle vie che facilmente collegavano Padova con le rotte adriatiche ${ }^{22}$.

\section{VERSO LE CIVITAS LAGUNARI}

Il percorso che porta dall'insediamento sparso (o comunque non nucleato) alla formazione delle civitas lagunari, dall'età augustea alla fine dell'età tardo antica, non è né lineare né unitario. Differenze locali, nelle modalità e nei tempi di aggregazione degli abitati, dipendono da diversi fattori. Tra questi, però, pare che la funzionalità di questi punti nodali tra entroterra e mare, prima che diventino veri e propri "empori", sia in gran parte da attribuire alla tipologia degli investimenti infrastrutturali fatta dai proprietari o dai gestori di tali aree di scalo. La trasformazione da semplici porti naturali a porti di interscambio, infatti, avviene grazie a complessi lavori di sistemazione portuale.

L'archeologia in anni recentissimi ha permesso di conoscere meglio alcuni luoghi chiave di questa trasformazione. A San Basilio, sul delta del Po, ad esempio, pare assodata la presenza di una struttura portuale di età imperiale, legata ad una stazione di sosta lungo la via endolagunare da Ravenna ad Aquileia. Il sito presenta una lunghissima continuità d'uso dall'età protostorica fino all'epoca alto medievale, quando diventa un "villaggio" di una certa grandezza. La presenza di edifici religiosi esprime l'importanza di una comunità saldamente presente nel territorio, forte di una maturata esperienza di traffici e gestione di spazi lagunari. La comunità deve la sua fortuna alle lavorazioni che si attivano nello scalo portuale. Lo stesso tipo di economia pare emergere dai recentissimi scavi presso la basilica di Santa Maria Assunta a Jesolo, in piena laguna alle foci del Piave: un abitato strutturato è qui presente fin dall'età imperiale e il numero delle persone che vi abita in età altomedievale (nel VI secolo) diventa tale da consentire la costruzione di un edificio religioso riccamente decorato ${ }^{23}$. Equilo-Jesolo, data la sua posizione, deve per forza essere interpretato come uno scalo portuale all'imbocco della via fluviale verso l'entroterra trevigiano.

Ma chi investe in queste infrastrutture portuali?

Un ruolo determinate per l'attivazione economica di questi scali deve essere stato svolto dalle élite aristocratiche legate agli ambienti militari. Ad esse si devono molte azioni volte all'organizzazione sociale e politica degli insediamenti lagunari delle origini. Tale ipotesi di lavoro si evincerebbe da due ordini di fattori: l'uno che discende da una semplice constatazione di tipo geografico, l'altro si desume da alcune fonti storiche.

Il primo dato da considerare è di ordine topografico e geografico. La tipologia dei terreni dove fisicamente i nuovi 
insediamenti prendono spazio, lo si è detto, è del tutto peculiare. Si tratta in gran parte di isole lagunari legate a sistemi deltizi di fiumi di una certa importanza. Barene sabbiose e dossi fluviali che hanno la caratteristica di costituire luoghi di possibile insediamento "solo" a partire dall'età imperiale, per il semplice motivo che prima (in età repubblicana) quasi non esistevano ${ }^{24}$. Terre nuove, dunque, e in corso di veloce formazione: ma non per questo "terre di nessuno". Si tratta di vaste aree con lagune e aree di basse acque, che entrano a far parte, naturalmente, nelle proprietà pubbliche del demanio imperiale.

Possiamo immaginare tali proprietà come dei saltus, grandi aziende imperiali che - ad esempio - sono attestate nell'area del delta del $\mathrm{Po}^{25}$. A Voghenza (Vicus Aventia) una di queste grandi aziende agricole di proprietà statale sembra avere nel vicus stesso il centro amministrativo e di controllo, come sarebbe testimoniato da una serie di epigrafi che ricordano servi, liberti e almeno un dispensator, che avrebbe gestito le proprietà fiscali dello stato ${ }^{26}$. Altre terre, di nuova formazione, possono essere state date come appannaggio a membri dell'esercito alla fine delle loro carriere, secondo una pratica comune nella tarda antichità. Tale pratica continua verosimilmente anche in età gota $\mathrm{e}$ bizantina: vengono così trasferiti a dignitari dell'esercito vaste proprietà. Proprietà, che come si è detto, contano campi, lagune e foreste. Proprietà che presuppongono un certo numero di servi, stanziati in loco, per la gestione delle terre (pratiche agricole), delle acque (pesca e salinatura), delle foreste (governo e taglio dei boschi).
Sappiamo che le proprietà fondiarie (e vallive) di area perilagunare tendono ad agglutinarsi in ampi latifondi tra II e V secolo. Cosi, almeno, pare essere avvenuto nell'area intorno a Cittanova ${ }^{27}$. Nel passaggio, poi, dall'età gota all'età bizantina, il ruolo di alcuni dei gestori di tali fondi inizia ad assumere connotati sempre più peculiari. Tribunie magistri militum, infatti, sembrano assumere sempre più la fisionomia tipica del grande proprietario fondiario: il loro ruolo politico - soprattutto nei confronti della popolazione agraria (e lagunare) che risiede all'interno delle loro proprietà - è in bilico tra funzioni pubbliche e diritto privato ${ }^{28}$. Grandi proprietà, dunque, appannaggio di aristocrazie terriere e, con buona probabilità, legate ad alte cariche dell'esercito.

Questo ruolo che oscilla tra pubblico e privato, tra funzionari militari e proprietari terrieri (di stampo pre-feudale) può essere attribuito ai tribuni presenti nelle epigrafi e nelle fonti scritte altomedievali, tra cui tra gli altri possiamo ricordare la nota iscrizione da Jesolo, che ci tramanda un Antonino tribuno ${ }^{29}$.

Dalla stessa Torcello vengono alcune indicazioni epigrafiche che fanno riferimento, fino a partire dalla tarda età imperiale, a servi e liberti che possono essere stati i protagonisti di questo spazio agrario/lagunare. Uno studio attento di questi materiali epigrafici ${ }^{30}$, in chiave antropologica più che cronologica, potrebbe aiutarci a ricostruire alcuni frammenti di tali società tardo antiche. Ciò che possiamo a buon diritto immaginare è che, legate a queste figure fortunosamente ricordate da sporadici documenti scritti, esistevano gruppi di persone che costituivano la parte

\section{Età Romana / Altino / Padova}

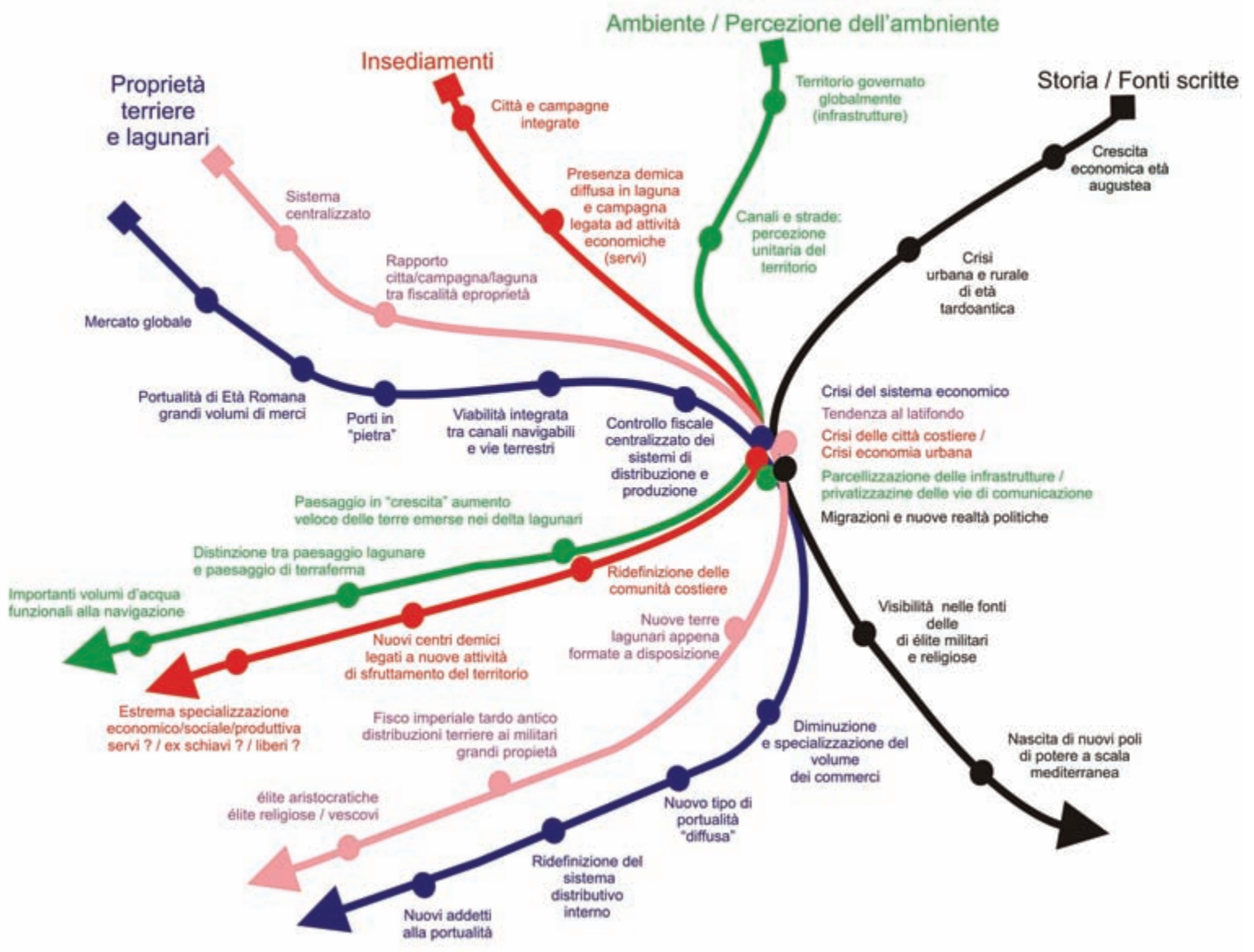

\section{Venezia e la Venetia altomedievale}

Fig. 1. La formazione degli insediamenti nella Veneta altomedievale. 


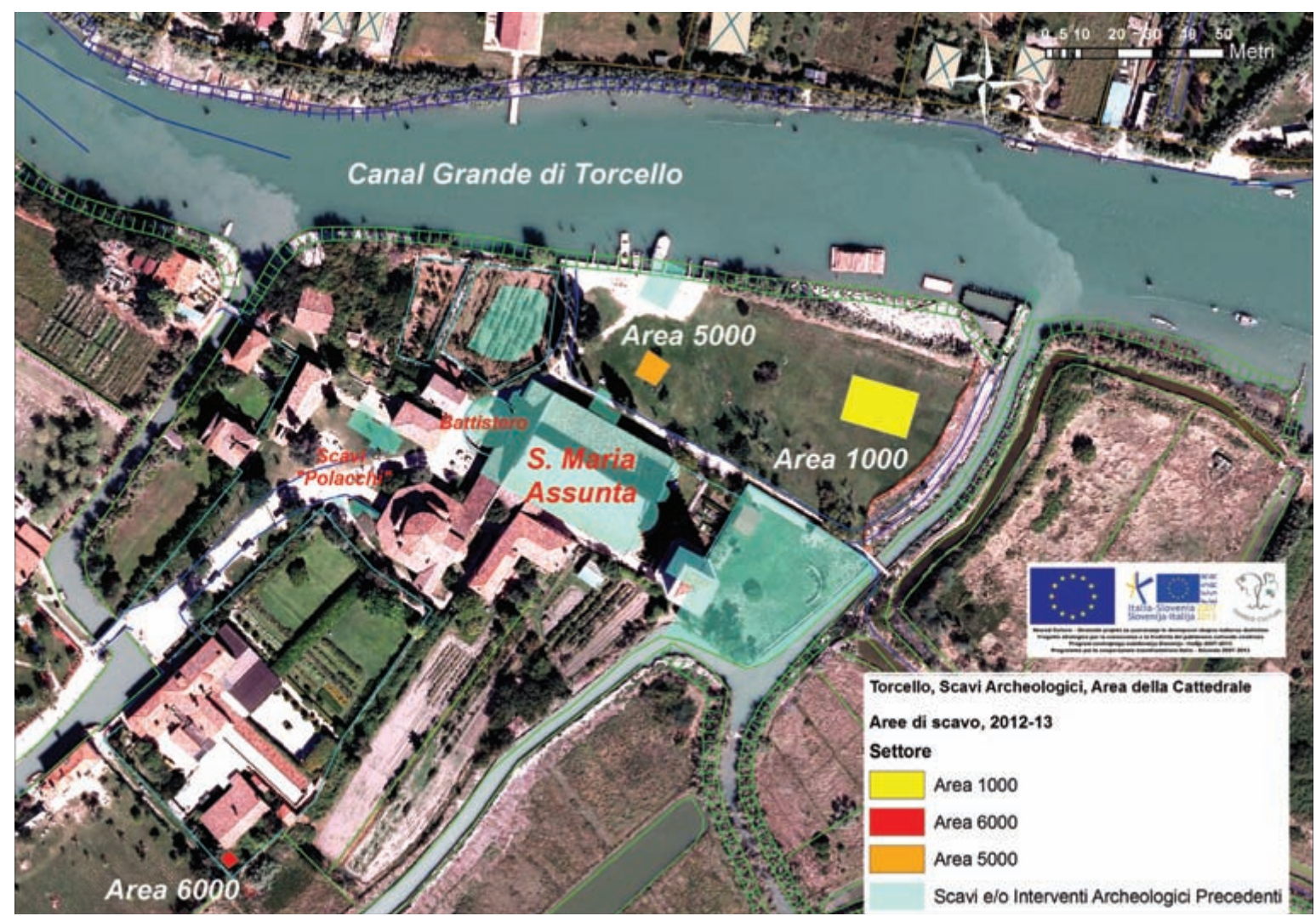

Fig. 2. Aree di scavo, Torcello 2012-23013.

"invisibile", ma numericamente più consistente degli habitatores della laguna. Questi, in un arco di tempo piuttosto lungo, attraverso un reciproco rapporto di interscambio e di adattamento con l'ambiente circostante, hanno potuto sviluppare competenze tecniche di alto livello legate alla marineria e alla produzione di navi. Elemento che costituirà uno scarto qualitativo di assoluta importanza nel momento in cui le autorità bizantine dell'area si disgregheranno e non riusciranno più a controllare efficacemente i flussi di traffico e la gestione dei punti di interscambio.

\section{ANTROPOLOGIA ED ECOLOGIA: LA LAGUNA E POPOLAMENTO OLTRE I CONFINI CRONOLOGICI}

Quale può essere, dunque, il ruolo e i metodi dell'archeologia che possiamo utilizzare per studiare l'adattamento umano (e le competenze tecnologiche che ne derivano) a questo ambiente? Come si può rileggere il complesso e vivificante rapporto tra gli uomini e la laguna nel lungo periodo, dalla tarda antichità fino all'alto medioevo? Quali sono gli elementi riconoscibili nel terreno che ci aiutano a definire materialmente gli spazi abitati e frequentati nel momento che precede la nascita di Venezia? Qual è poi, il contesto di riferimento - sia su scala regionale che su scala europea del fenomeno?

Queste pagine, sollecitate dal coinvolgimento e dall'apertura di in un nuovo grande scavo lagunare a Torcello (cfr. oltre): tornano a riflettere (purtroppo, ancora una volta!) sulla problematica delle origini e, per quanto difficile, tentano di affidarsi il più possibile ai dati materiali (fig. 01).

Il punto di partenza, interpretativo e di metodo, è costituito dagli apporti nel dibattito storico di un'impostazione piuttosto recente. Si tratta dell'approccio che fa uscire la genesi degli abitati lagunari dalle dinamiche meramente locali e porta Venezia (con Comacchio) all'interno del dibattito sulla formazione di un mercato altomedievale a scala europea. Tale impostazione ha informato i lavori di un fortunato congresso tenutosi a Comacchio nel 200931, in cui si sono presentati i risultati degli scavi comacchiesi, frutto di un progetto integrato e multidisciplinare. Il progetto è stato governato da una strategia che prevede il ricongiungimento della laguna con il suo entroterra, nell'ottica della lunga durata, rivedendo vecchi scavi, analizzando le sequenze di nuovi interventi e basandosi su indicatori commerciali e ambientali che sono effettivamente testimoniati nel terreno. Il parallelo con gli empori dell'Europa del nord, infine, permette uno stacco metodologico importante, che fa superare un'ottica localistica e, soprattutto, un'ottica storica ingessata su poche fonti antiche e su un'immensa mole di studi critici elaborati su queste stesse fonti.

L'occasione specifica per tornare sull'argomento, come si è anticipato, è offerta da un recente scavo archeologico in laguna: uno scavo presso la basilica di Santa Maria Assunta di Torcello, realizzato nel suo primo lotto di interventi nell'inverno del 2012-2013 (fig. 02). I risultati dello scavo, che ha portato alla luce alcuni settori dei quartieri abitativi altomedievali, stimolano nuove considerazioni sulla sequenza archeologica dell'isola. Uno degli obiettivi dello scavo è quello di considerare in prospettiva antropologica ed ecologica la "consistenza" di questi abitati lagunari. L'idea, non priva di rischi, è quella di valutare la percezione che in antico si aveva dell'ambiente lagunare, mettendo gli uomini e le donne del passato (soprattutto le masse di lavoratori della laguna nel loro divenire politico e sociale) al centro dell'attenzione. Ci si vuole chiedere, ad esempio, quanto spazio ogni unità abitativa abbia occupato in passato, quale sia stato l'impatto ecologico nel corso del tempo di tali abitati, quali siano state le relazioni sociali che si sarebbero sviluppate nella peculiarità dell'ambiente lagunare.

Se si dovesse riassumere quale sia stato fino ad oggi l'approccio ecologico che storici e archeologi hanno utilizzato per definire le caratteristiche dell'insediamento 
altomedievale, dovremmo per forza evidenziare come le azioni sul paesaggio (le modificazioni) siano state analizzate in chiave positivistica. Le comunità lagunari sono state descritte come forze "creatrici" della natura stessa. Il paesaggio delle origini è divenuto, sia nei racconti mitici della fondazione, sia nelle aggiornate ricostruzioni storiche degli ultimi anni, un paesaggio culturale. Un paesaggio storico dove una serie di azioni umane avrebbero apportato trasformazioni e miglioramenti: l'uomo avrebbe tramutato le isole inospitali della laguna in una sorta di idilliaco rifugio. Il paesaggio diventa "manufatto", totalmente artificiale, e diviene l'oggetto stesso dello studio storico. Un approccio, invece, che consideri le azioni umane (e dunque le scelte insediative) come conseguenza della natura del luogo un approccio non lontano da quello che generalmente si applica per spiegare le strategie di popolamento pre-classico - non ha mai avuto un forte rilievo, poiché l'idea della migrazione forzata in laguna per motivi politici e bellici, si è sempre imposta. Ma forse, ciò che è mancato di più, è un approccio integrato, di tipo ecologico, che tenti di studiare l'uomo all'interno dell'ambiente naturale, evidenziando i vicendevoli e complessi legami che hanno permesso la nascita di un ecosistema profondamente antropizzato.

Il progetto su Torcello, dunque, vuole tentare un metodo ecologico e antropologico che parta dai dati materiali. Ecco dunque l'esigenza di misurare l'estensione delle foreste, la quantità di materiale da costruzione che era possibile estrarre da quelle foreste, la quantità di combustibile che quei boschi potevano fornire: parametri che possono definire l'attrattiva insediativa delle lagune venete. Allo stesso modo, riuscire a valutare come sia avvenuta la deforestazione di ampie aree lagunari e perilagunari, costituisce un altro termine di raffronto per la definizione del senso ecologico degli antichi venetici. Si vuole tentare di analizzare il rapporto con la variazione delle batimetrie, con la presenza di acqua navigabile nei canali, con le strategie di approvvigionamento del cibo, eccetera..

Abbandonando la classica dicotomia tra entroterra longobardo e laguna bizantina, tra mondo germanico e mondo latino, tra episcopati lagunari e diritti ecclesiastici del clero dell'entroterra, la laguna assume le caratteristiche di un territorio ricco di opportunità.

Ma come possiamo studiare il livello di auto-consapevolezza ecologica dei primi Venetici nella laguna (governo delle foreste, governo dei flussi marea)? E ancora come possiamo studiare e valutare le implicazioni sociali che sul lungo periodo hanno determinato quelle precise pratiche di sfruttamento dell'ambiente lagunare (organizzazione del lavoro, etica politica, coscienza sociale)? Come ha funzionato il trasferimento tecnologico che permise ai Venetici di VIII secolo di sviluppare un arsenale in grado di produrre vascelli che solcavano più volte all'anno (con una regolarità pari a una moderna compagnia di navigazione) l'intero $\mathrm{Me}$ diterraneo? Date le caretteristiche dall'ambiente lagunare (insularità), quali sono stati i riflessi sociali dei meccanismi per assicurarsi il cibo (carne e vegetali)? Come si è risolto, e con quali conseguenze sul piano demografico, il problema dell'approvvigionamento di acqua dolce? Quali sono state le conseguenze sociali e di organizzazione del lavoro prodotte dallo sviluppo delle produzioni ad alta tecnologia (sia che si parli di una galea, che di un calice in vetro altomedievale)? Come riconciliare le narrazioni storiche sulle origini con un meccanismo economico basato sulla vendita e sul commercio, utilizzando il lavoro servile e commerciando, tra le altre merci, anche molti schiavi?

\section{ARCHEOLOGIA ED ECOLOGIA: DA ALTINO A TORCELLO (SENZA PASSARE PER ATTIILA)}

La storia archeologica di Torcello è emblematica. Il sito, scavato stratigraficamente più di 50 anni fa, ha offerto da lungo tempo agli studiosi materiali per ridefinire il "processo genetico" degli insediamenti legati alla laguna di Venezia ${ }^{32}$. Gli scavi dell'Accademia Polacca di Scienze avevano chiarito in maniera definitiva che l'insediamento dell'isola non era da attribuire ad un'unica ondata di migrazioni, che esisteva una precedente occupazione di età romana, che il tipo di strutture rinvenute indicava un certo investimento in infrastrutture (rialzi, bonifiche, ecetera) che non era compatibile con un' occupazione di tipo marginale e/o temporanea ${ }^{33}$. Questi scavi sono stati seguiti da molti altri interventi archeologici, di diversa natura, ma che - in linea generale - hanno ribadito questa linea interpretativa, volta a riconoscere (pur con alcuni vuoti stratigrafici per taluni secoli) una sorta di continuità di occupazione, con usi differenziati della "barena" che oggi conosciamo come Torcello $^{34}$. L'archeologia, in buona sostanza, nonostante l'oggettiva problematicità di raggiungere per ampie aree le stratigrafie più antiche (poste a quasi due metri di profondità dal piano di campagna e permanentemente al di sotto dei livelli medi di marea) ha indicato che l'isola ha avuto nel tempo funzioni e occupazioni diversificate. A partire dall'età imperiale si riconosce una prima frequentazione (una villa marittima ?), proseguendo nell'età tardo imperiale, distinguendo un periodo di criticità tra III e V secolo (pochissimo o quasi per nulla rappresentato dai materiali ceramici, anche residuali), documentando una ripresa stabile dell'insediamento e dell'occupazione dal VI secolo in poi (con strutture in muratura, con case, con moli), fino a tutto l'alto-medioevo (con zone artigianali, aree produttive, edifici religiosi). Se invece che a Torcello (così prossima a Venezia da esserne adombrata) questi dati fossero stati rinvenuti in un sito presso il Po o, comunque, in un'area lagunare non così caratterizzata sul piano delle aspettative per la storia della genesi della Serenissima, la loro interpretazione sarebbe risultata piuttosto lineare. Nessuno (né archeologi, né storici) avrebbe avuto problemi nel definire Torcello un sito costiero/lagunare in evoluzione, almeno dalla tarda età imperiale in poi, profondamente legato al suo entroterra e agli insediamenti coevi, caratterizzato da un certo floruit insediativo collocabile alla fine del VII secolo e VIII secolo, testimoniato da una buona disponibilità economica tale da permettere l'erezione di un edificio religioso di importanti dimensioni. Un sito normale, dunque.

Torcello, invece, è stata interpretata in chiave evoluzionistica, come prodromo di Venezia. Così gli archeologi: si tende infatti a leggere nelle strutture abitative in legno o nel ricorso al riuso di materiali edilizi un segno di "fragilità" e di modestia legato ai bisogni locali di immediata sopravvivenza, comprovando l'idea della fuga. Pur riconoscendo la vitalità economica, palesemente dimostrata dalla quantità e dalla qualità dei reperti anforici e ceramici di importazione e provenienti da tutto il Mediterraneo, non si riesce a immaginare il sito come stabile se non nel momento in cui la massima autorità civile e religiosa (il vescovo) non decida di trasferirvisi, rifondando una nuova Venetia $^{35}$. Così gli storici: anche chi ha riservato una grande attenzione alle fonti archeologiche, pur riconoscendone l'evidenza, nel momento dell'interpretazione mette in gioco un'idea preconcetta che fa immaginare gli insediamenti più antichi come collocati in uno spazio dalle condizioni 
climatiche "difficili", segnato dalla "durezza del rapporto con l'acqua”, posto al di là del confine "minaccioso" tra provincia lagunare e regno longobardo, con una popolazione che avrebbe vissuto con una mentalità "da sfollati in attesa di un rientro" 36 .

Torcello non è Venezia, e non lo sarà mai. Torcello non si evolve in Venezia, semmai (in senso demico) si evolve in Burano medievale e Treporti di età basso medievale e moderna. E' uno dei luoghi che entra fin da subito nel dominio Veneziano, mantenendo però la sua identità per lungo tempo. Identità segnata da una distinta Diocesi e da una distinta percezione, anche in antico, dell'isola rispetto a Rialto e Venezia. Anche in epoca moderna i resoconti dei viaggiatori e le fonti scritte documentano come questo luogo sia sempre percepito come "altro" rispetto a Venezia. La stessa cronachista che celebra le mitiche origini veneziane, tende a non includere Torcello nel processo genetico. La fonte più autorevole, Giovanni Diacono, cita Torcello tra i luoghi della Laguna, ma l'isola non partecipa alla costruzione dell'antichità di Venezia ${ }^{37}$.

Torcello, se proprio dobbiamo assimilarla a qualche altro sito, è, piuttosto, "Altino". Il Vescovo altinate stesso, che qui si fa costruire la sua cattedrale, non sente la necessità di cambiare nome al suo titolo, e si firma nei documenti altomedievali come "vescovo di Altino". Ma non si tratta solo della titolatura della cattedra episcopale: Torcello, da quanto ci dice l'archeologia, si configura come un'area periferica della città romana di Altino. Tale area tra tarda antichità e alto medioevo assume più importanza della città romana (che è in grave crisi economica e politica), tanto che la massima autorità religiosa avrebbe scelto di trasferire la sua sede laddove già molti cittadini avevano progressivamente trasferito (nel corso di più generazioni) interessi economici, residenze e i loro servi e schiavi ${ }^{38}$.

Altino, città emporiale adriatica, è una città romana di tipo lagunare. Altino era connessa agli altri porti dell'alto Adriatico tramite i canali e i delta fluviali. Le aree di snodo di tali rotte navigabili costituivano spazi di notevole interesse logistico, e necessitavano di basi e approdi per una portualità che, in età tardo imperiale, tende a diventare "diffusa". Come in altre aree dell'alto Adriatico (si pensi a Grado, ad esempio), dall'età tardo imperiale in poi si assiste al passaggio da un unico grande porto controllato (anche fiscalmente) dall'autorità imperiale, alla dislocazione dei servizi portuali in siti satellite, collocati lungo le nuove rotte navigabili fluviali e, verosimilmente, controllati da nuove generazioni di investitori e armatori. C'è il sospetto che tale portualità sia legata ad investimenti specifici, non necessariamente pubblici (o almeno non totalmente pubblici). A tale portualità possono essere connesse le evidenze legate allo sfruttamento delle risorse, sale e itticultura e, dove la terra lo permette, coltivazioni orticole e agricole ${ }^{39}$.

I porti tardo antichi sono molto diversi dai porti di età repubblicana e imperiale ${ }^{40}$ : la contrazione economica e una differente circolazione delle merci fa si che le città litoranee abbiano una minore disponibilità di materiali provenienti dalle cave. I moli e le banchine in pietra, così, vengono sostituiti sempre più spesso da moli e banchine in legno.

A Lio Piccolo, in prossimità di Torcello, qualche anno fa è emersa una grande cisterna, dotata di una canna da pozzo per immagazzinare dell'acqua: era una riserva di acqua dolce lungo una via navigabile e destinata al servizio delle navi di passaggio ${ }^{41}$. Numerose sono le basi a forma quadrata o pseudo rettangolare che sono state interpretate come basi per torri faro, strutture a supporto della navigazione ${ }^{42}$.
Certamente a Torcello e nei dintorni dovevano esserci elementi infrastrutturali per la statio delle imbarcazioni (con cavane, porti secondari, ma anche con tabernae e edifici di ricezione). Non potevano mancare i magazzini e zone attrezzate per il ricovero e la riparazione dei natanti (arsenali). Nei percorsi endolagunari, inoltre, molti dovevano essere i luoghi che richiedevano infrastrutture speciali, come ponti mobili e zattere per il trasbordo di canali ${ }^{43}$.

Future indagini archeologiche potranno descriverci meglio la topografia della laguna in età tardo romana che, tra le altre cose risulta di non facile lettura per la fragilità dei rinvenimenti (legno, fango), per la necessità di operare spesso in scavi subacquei (logisticamente più complessi degli scavi terrestri) e, non da ultimo, per una mancanza di strategie rivolte al recupero e studio delle strutture in materiale deperibile. Oggi, però, si è in grado di dire che la laguna (e in speciale modo la laguna nord) costituiva l'estensione al di fuori dei confini strettamente urbani (extra pomerium) di Altino, una sorta di contado acqueo.

Una laguna abitata, dunque. Abitata da servi e schiavi che sono impiegati nella costruzione delle banchine, dei porti, dei magazzini. Dal personale che serve per lo scarico e il carico delle merci. Dagli artigiani che sono di supporto alle attività cantieristiche. Un paesaggio popolato da molti lavoratori e da pochi membri di una élite di investitori e gestori (commerciali, portuali, agrari, salinatori etc.).

Non vi è alcun buon motivo per immaginare che chi lavorasse in peschiere, in saline o in scali portuali vivesse nell'area di Altino e, giornalmente, si recasse nei luoghi di lavoro. E' più credibile pensare che si siano formati dei quartieri per i lavoratori, in alcuni casi anche stagionali, ma che ben presto con il progressivo aumentare dei volumi di traffico hanno assunto caratteristiche di un insediamento stabile.

Numerosi interventi archeologici hanno chiaramente dimostrato come la città romana di Altino sia andata incontro già a partire dal II-III secolo d.C. ad un crisi interna, di tipo portuale: moli defunzionalizzati, canali insabbiati, banchine riadattate e vie d'acqua colmate ${ }^{44}$. Tali cambiamenti sembrano potersi ascrivere più a mutate situazioni topografico-ambientali (cambiamenti geografici legati all'orografia dei fiumi locali) che a distruzioni e guerre. La città, infatti, continua a essere un luogo di attrattiva nella tarda antichità, ma pare perdere settori di portualità interna. Manca ancora uno scavo sistematico del porto vero e proprio, individuato da recenti e interessantissime attività di telerivelamento ${ }^{45}$, che potrebbe confermarci se la situazione altinate possa essere effettivamente assimilata al destino del porto di Ravenna, interrato nel III secolo d.C., come è riportato dal famoso passo di Iordanes ${ }^{46}$.

Con l'interramento dei canali non solo si assiste alla perdita delle caratteristiche forme urbane, ma la mancanza delle vie d'acqua sottrae ad Altino la sua funzione primaria, e cioè quella di porto.

Le lagune si popolano, o meglio, vengono forzosamente popolate in seguito alle strategie economiche delle élite tardo antiche. Oggi non possiamo sapere attraverso i resti di pasto o i frammenti dei contenitori da cucina quale sia la condizione giuridica dei lavoratori in laguna, se servi, liberi o semiliberi ${ }^{47}$. Possiamo, però, ipotizzare che il Vescovo di Altino, abbia scelto di posizionare la sua sede in un quartiere periferico, ma assai vivo, forse più popoloso dell'antico centro.

Ma facciamo un passo indietro. 


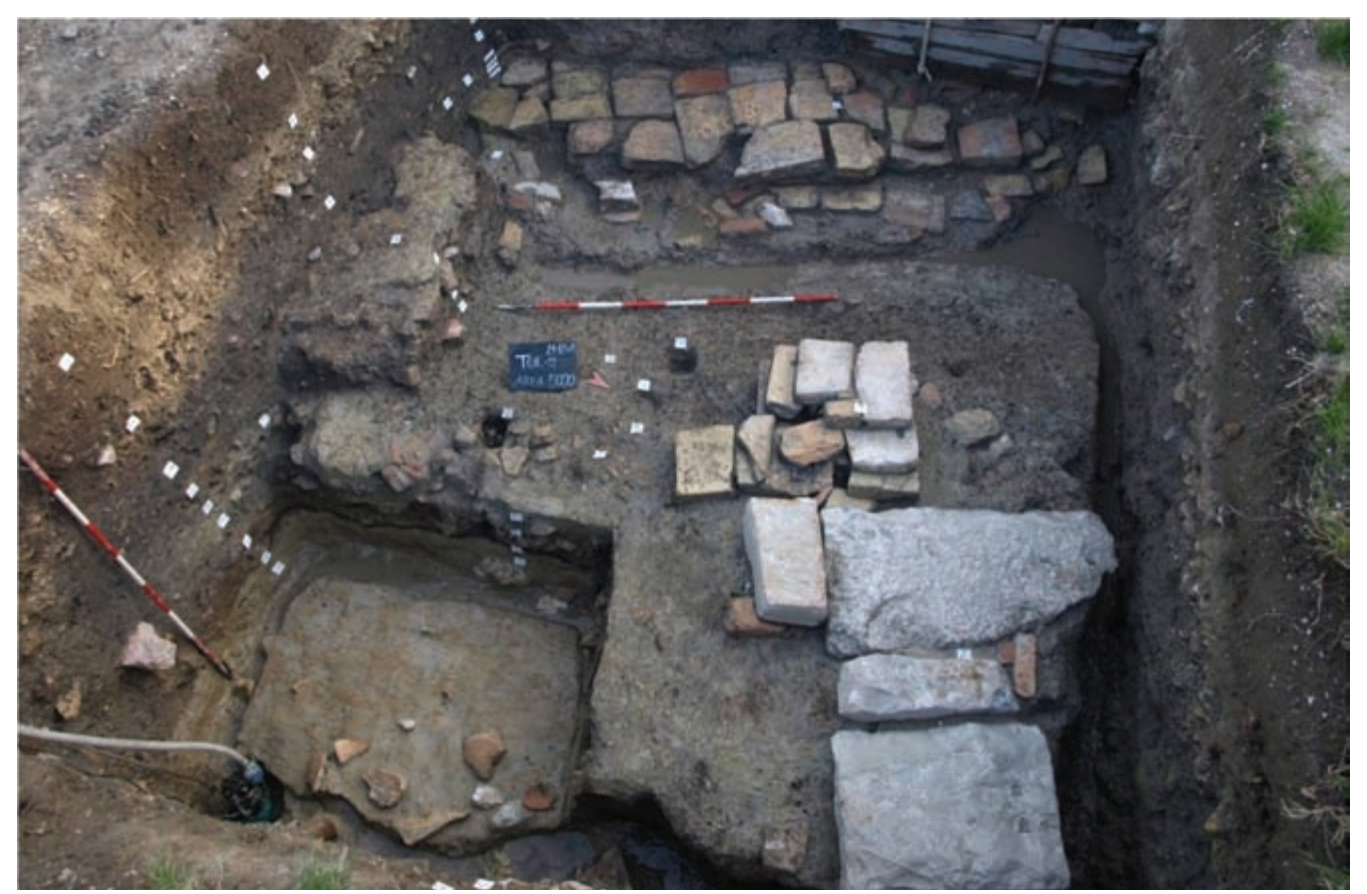

Fig. 3. Torcello 2012-2013, area 5000: Porzione di magazzino di VI-VII secolo.

Le sequenze dello scavo del 2012-2013 a Torcello mostrano una frequentazione dell' arcipelago in età imperiale. Il contesto più antico rinvenuto è legato ad un'attività di sistemazione di un argine (waterfront), in vita tra la seconda metà del I secolo a.C. e tutto il I sec. d.C.. E' una riva di contenimento, rinforzata da pali verticali, con un massiccio riporto di argille irrobustito dall'uso di materiali ceramici (eminentemente frammenti di grandi anfore) e laterizi, usati come materiale compattante e drenante. Il tipo di riporto richiama lo strato VIII emerso negli anni '60 del secolo scorso nello scavo dell'équipe polacca ${ }^{48}$. Situazione simile è stata messa in luce negli scavi più recenti al di sotto delle aree del Battistero e della Basilica ${ }^{49}$. In entrambi i casi le attività di sistemazione sono legata alla creazione di un riporto, che sottende la presenza di un edifico "interno", collocabile probabilmente nell'area dell'attuale Basilica. L'esiguità dell'estensione dello scavo non ci permette che ipotesi sulla natura di tale struttura. Non sono poche, però, nell' area le attestazioni di rive, moli (definiti a volte impropriamente "argini strade") con una cronologia collocabile tra il I e il II sec. d.C.50. Si tratta di banchine per il carico/ scarico di merci? Si tratta di strutture collegate alla conterminazione di aree per lo sfruttamento delle risorse lagunari (vasche per itticultura o salinatura)? Questi elementi (sale, pesce, portualità) devono per forza essere i motori economici delle note "ville" marittime presso Altino, ricordate nel celebre passo di Marziale ${ }^{51}$.

La sequenza del 2012-2013 (ma anche gli scavi degli anni passati) non fornisce dati confortanti per il III e la prima metà del IV secolo d.C.: rari materiali ceramici ${ }^{52}$ anforici ${ }^{53}$, vitrei ${ }^{54} \mathrm{e}$, soprattutto, mancanza di strutture edilizie di rilievo. Non possiamo ancora dire se vi sia frequentazione e di che tipo, se si assiste ad una contrazione demica frutto di una crisi ambientale o economica. Le crisi potrebbero essere legate allo specifico ambiente dell'isola o a condizioni di economia regionale ${ }^{55}$.

Lo scavo del 2012-2013, per i secoli successivi (fine VIVII secolo) fornisce importanti elementi (scavo area 5000), che portano ad immaginare la presenza di un area di magazzini all'esterno della Basilica. Siamo presso l'area della quarta navata, leggermente spostati verso nord rispetto alla collocazione degli scavi diretti da Maurizia De $\operatorname{Min}^{56}$. Qui si è scavata la porzione di un edificio con fondazioni in mattoni legati da argilla. Era direttamente affacciato sul canale e aveva un portico in facciata con pilastri quadrangolari. L'edificio era costruito in mattoni romani di riuso. I piani si sviluppavano verso l'attuale Basilica. All'esterno, verso il canale, i plinti quadrati, poggiavano su grossi calcari d'Istria e marne di riuso, costituenti una banchina in pietra. Il muro non era legato da malta, forse era in tecnica mista, ma aveva quasi certamente un certo sviluppo in altezza, testimoniato dall'ampiezza delle fondazioni, dalle buche pontaie e dai plinti in mattoni (fig. 03).

L'edificio ha un confronto stringente con i magazzini scavati nel porto di Classe, ad esempio le strutture intorno al cosiddetto "magazzino n. 17". A Classe il magazzino presenta un portico esterno su pilastri del tutto simile a quello rinvenuto a Torcello. La posizione sul canale e la larghezza del portico, qualificano la tettoia come non destinata al "soggiorno" (e quindi legata ad un abitazione) ma funzionale allo scarico e carico dei natanti attraccati alla riva. Tra le altre cose, inoltre, anche a Torcello una strada, datata al VII secolo, sembrerebbe essere stata ritrovata durante l'intervento di scavo presso la quarta navata.

I dati di scavo ci portano a pensare che le note case con zoccolo in muratura, identificate e proposte nella ricostruzione dello scavo degli anni ' 90 del secolo scorso ${ }^{57}$, in realtà siano, per ragioni stratigrafiche, reinterpretabili e assimilabili a tali magazzini. L'attribuzione abitativa di quelle strutture deriverebbe dal fatto che in una fase successiva (fine VII secolo, inizi dell'VIII) il magazzino viene demolito e, sopra di esso, si costruiscono abitazioni in legno, con pali angolari e alzato in materiale deperibile. Queste abitazioni, con più fasi di vita per almeno un secolo, sono caratterizzate da battuti di argilla e focolari a terra. Lo scavo del 2012-2013, sembra evidenziare uno scarto cronologico e stratigrafico, con un evidente taglio, tra questi piani pavimentali e l'edificio in mattoni.

Le stesse abitazioni in legno sono state individuate anche in un'area piuttosto lontana dalla chiesa (area 6000), presso l'ex scuola elementare. Nell'area 1000 si è potuto indagare un settore coevo di un'area produttiva. Si tratta 


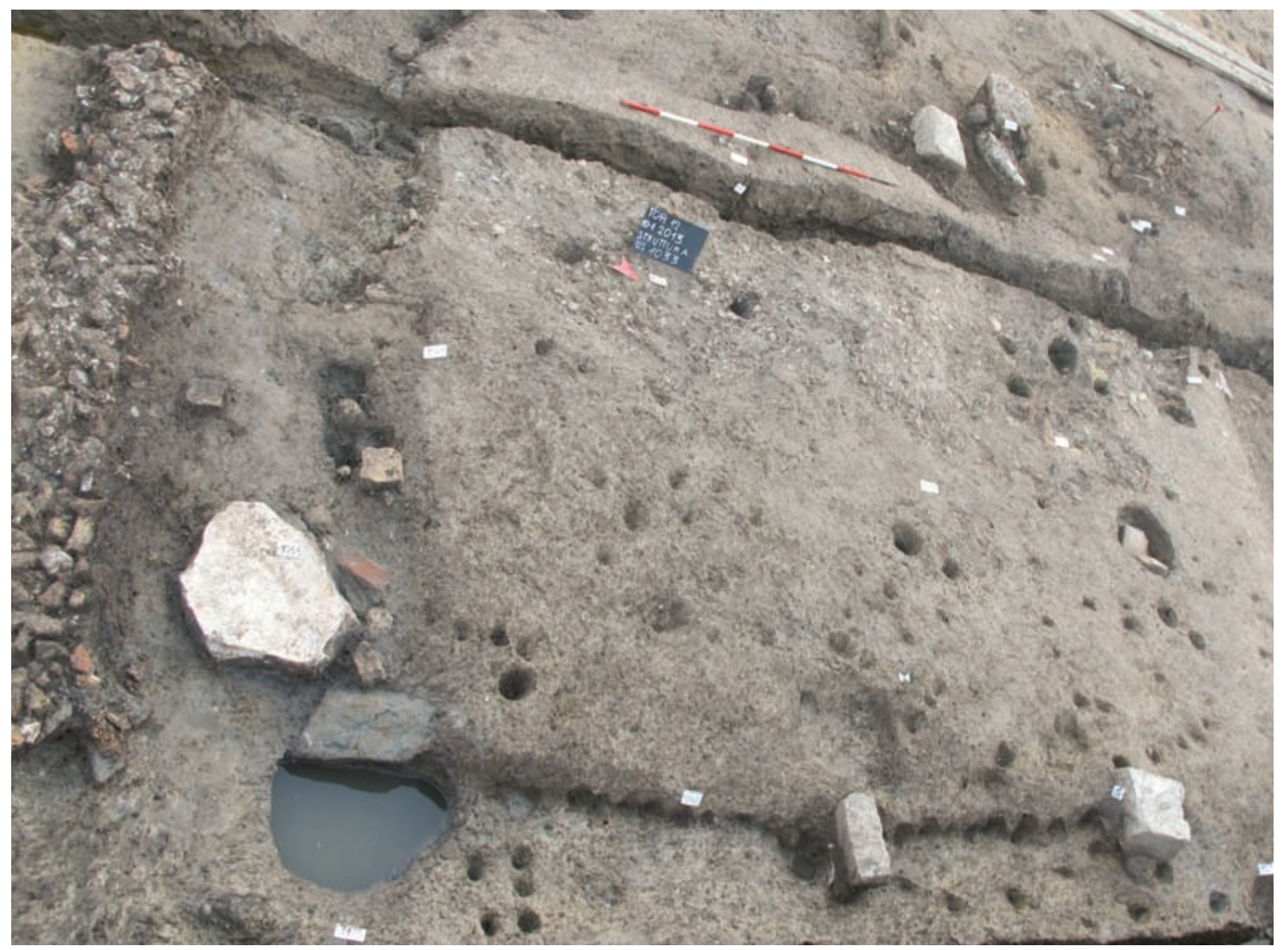

Fig. 4. Torcello 2012-2013, area 1000: Edificio abitativo in legno di IX-X secolo.

della base (tagliata dalle fasi successive) di una piccola fornace, probabilmente una fornace secondaria, a forma rettangolare, con fondazioni in frammenti di laterizi. Si è conservato un ampio strato di ceneri e carboni, spesso fino a $15 \mathrm{~cm}$, in corrispondenza di quella che doveva essere la bocca di alimentazione. La struttura, coperta da una tettoia in legno, pare essere stata restaurata almeno un paio di volte, per essere poi completamente rasata prima di impiantare le abitazioni della fase successiva. La setacciatura completa delle unità stratigrafiche del complesso e, soprattutto, di quelle relative agli scarichi di cenere e carboni, porta ad ipotizzare un'area per la lavorazione del vetro. La dimensione e il ritrovamento di un piede di calice fallato (tipo Isings $116 / 117^{58}$ ), insieme ad alcune goccialature di vetro fuso, fa immaginare l'uso del forno per l'ultima fase del ciclo produttivo del vetro, ovvero quella relativa alla ricottura dei prodotti forgiati. Se l'interpretazione è corretta, una fornace principale (per la fusione e la soffiatura) doveva essere collocata non troppo lontano. Tale area produttiva confermerebbe l'idea di una vocazione artigianale per la produzione del vetro a Torcello. Nasce l'ipotesi che vi siano più botteghe dislocate nell'isola, che immetterebbero nei circuiti commerciali prodotti non dissimili da quelli documentati nella celebre fornace scavata begli anni ' $60^{59}$.

Queste attività non sembrerebbero le uniche attività artigianali presenti: semilavorati in osso fanno ipotizzare che esista un'industria locale per la lavorazione di pettini e, in genere, di manufatti, sia di uso domestico che artigianale ${ }^{60}$.

Al di sopra delle fasi artigianali si è scavata una porzione di un quartiere abitativo. L'area era densamente abitata, con case organizzate intorno a un cortile centrale, una sorta di campiello. Il "campo" era provvisto di un grande sistema di approvvigionamento idrico, ovvero una cisterna-pozzo

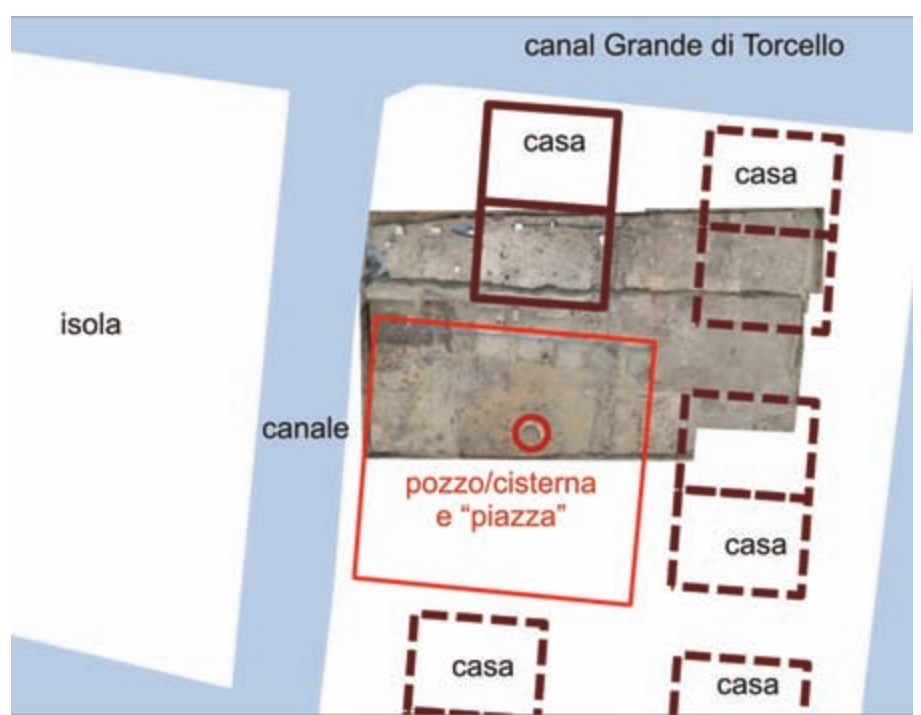

Fig. 5. Torcello 2012-2013, area 1000: Schema ricostruttivo della disposizione degli edifici abitativi intorno alla cisterna-pozzo.

alla veneziana. La canna del pozzo era realizzata completamente con materiali di recupero di età romana. La vita di questo quartiere si colloca tra IX e X/XI secolo (fig. 04 e 05).

Intorno al pozzo sono state portate alla luce le strutture in negativo (le buche di palo, le spoliazioni e le fondazioni) delle case. Il settore abitativo è caratterizzato da un'organizzazione piuttosto regolare degli spazi. Sul campo si affacciano a distanze regolari le case di legno, unifamiliari, caratterizzate da un'edilizia di alta qualità. Edifici probabilmente a due piani, a quattro vani, di cui i due inferiori sono dedicati alle attività di magazzino e ospitano un focolare. I fuochi, costruiti a terra, sopra riporti di argilla isolante, 


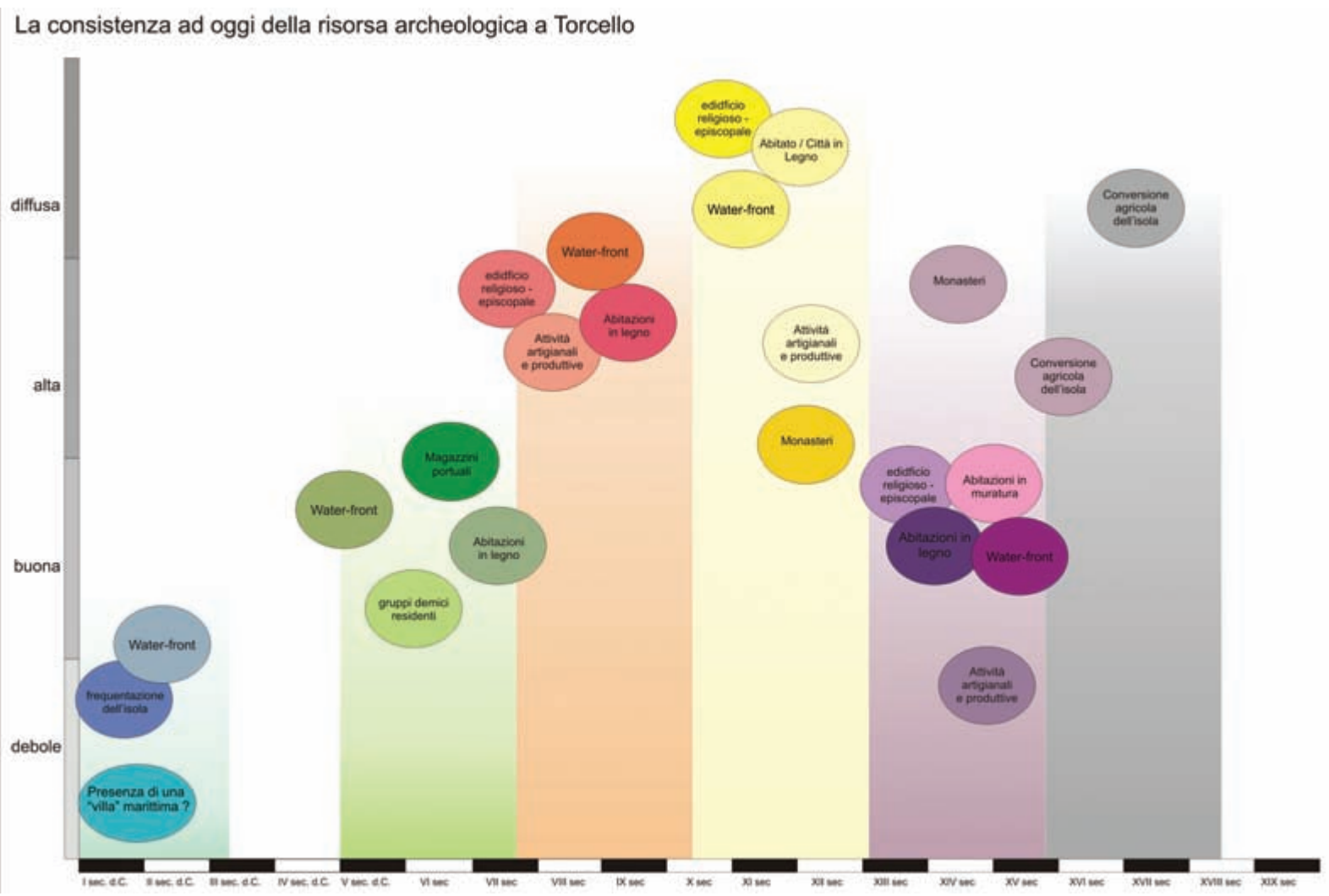

Fig. 6. Torcello, schematizzazione della risorsa archeologica nota fino ad oggi.

servivano per la cottura dei cibi e per il riscaldamento. Al piano superiore, probabilmente, vi erano le camere destinate alla famiglia.

Tra un edificio e l'altro vi sono cortili, ben distinti, funzionali alle attività da svolgere all'aperto. Ogni edificio, inoltre, si affaccia sul canale e possiede una sorta di porta o accesso d'acqua: l'unica via di comunicazione tra un settore e l'altro della città, poiché non esistono strade (né ponti) sono i canali. Possiamo definire queste abitazioni come una sorta di prototipo delle case "fondaco", tipiche dei mercanti della Venezia medievale.

Il legno, dunque, è l'elemento caratterizzante del paesaggio altomedievale e medievale lagunare. Non soltanto per $\mathrm{i}$ boschi e le foreste che all'epoca ricoprivano fittamente le barene circostanti, ma perché il legno diventa il materiale costruttivo per eccellenza. L'archeologia ha potuto documentare situazioni analoghe a Cittanova, a Rialto, a Comacchio: il legno è attestato nella costruzione di waterfront, rive, moli, approdi e per l'edilizia civile. Case, botteghe artigiane e ricoveri per animali: ogni edificio ha alzati in legno. Pochi sono gli edifici costruiti con laterizi e pietre (tutti materiali di reimpiego): si tratta per lo più di edifici religiosi e pubblici.

Gli edifici di legno, comunque, corrispondono a strutture abitative piuttosto complesse: il legno costituisce una risposta tecnologica pertinente ad un ambiente del tutto peculiare come quello lagunare. Il legno è più leggero e ha un maggior potere isolante, e meglio si adatta alle caratteristiche anfibie del luogo.

Non solo Torcello, ma tutte le città lungo le lagune erano eminentemente in legno: tanto che, come giustamente è stato osservato, i centri altomedievali dell'alto Adriatico assomiglierebbero di più agli empori/città carolingi dell'Eu- ropa settentrionale che alle città bizantine del Mediterraneo ${ }^{61}$. La quantità di legno necessario (e la forza lavoro) per tutte le lavorazioni è incredibilmente alta, tale da richiedere una chiara politica di gestione delle foreste. Per dare un esempio numerico, utilizzando il porto di Comacchio di VIII secolo, si consideri che in un settore scavato presso il Villaggio San Francesco ${ }^{62}$, si sono indagati 16 mq di pontile/banchina, con il rinvenimento di circa 25-30 grandi pali, corrispondenti a loro volta a 15/20 alberi abbattuti. Si è calcolato che il porto di Comacchio abbia avuto una estensione massima di $75.000 \mathrm{mq}$. Se ammettessimo che il porto (compresi i magazzini lignei) sia stato realizzato nell' arco di un anno, dobbiamo ipotizzare l'abbattimento di circa 110.000-120.00 alberi, ovvero circa 300 alberi al giorno.

Ma il legno è il materiale da costruzione anche di altri elementi essenziali del panorama altomedievale di Torcello: imbarcazioni, zattere, ponti e pontili.

I dati di scavo, qui presentati, sono chiaramente di tipo preliminare, e andranno verificati nel corso delle prossime campagne (fig. 06). Il progetto, però, si svilupperà, per quanto possibile, all'interno della stessa linea interpretativa, con l'intento di meglio comprendere fino a che punto l'acqua e la gestione delle risorse ambientali abbia creato quello specialissimo legame tra uomo e ambiente, che costituisce la peculiarità delle comunità veneziane. Si tratta di un legame reciproco e complesso, dove un ambiente apparentemente limitante ha permesso la creazione di specifiche competenze tecniche e distinte strutturazioni sociali. In un momento preciso della storia dell'alto medioevo padano e adriatico tali competenze sono risultate vincenti nell'attirare interessi economici in grado di cambiare i destini del mercato mediterraneo e europeo. 
${ }^{1}$ Il presente testo propone le linee di metodo del progetto The Voices of Venice: Anthro-Ecological Perspective on the Making of Medieval Europe, EU - Marie Skłodowska-Curie IOF 2013, Università di Stanford (USA) - Università Ca' Foscari, Venezia. Il progetto costituisce uno spin-off dello scavo archeologico iniziato nel 2012-2013 nel quadro di un progetto Interreg Italia-Slovenia Shared Culture, promosso dalla Regione Veneto in collaborazione con l'Università Ca' Foscari. La direzione scientifica per la parte archeologica era affidata a chi scrive, in collaborazione con il prof. Guido Biscontin e Elisabetta Zendri. L'ideazione e la coordinazione del progetto è stata a cura della Dr. Clara Peranetti (UCPSPC), l'organizzazione tecnica e logistica è stata coordinata Geom. Daniele Scatto (Genio Civile), entrambi della Regione Veneto. Il testo che segue rielabora in parte e in forma sintetica i dati contenuti nelle pubblicazione di progetto dedicate a tale scavo: D. CALAON, E. ZENDRI, G. BISCONTIN (a cura di), Torcello scavata, patrimonio condiviso. Lo scavo 2012-2013, Venezia, 2014; D. CALAON, Quando Torcello era Abitata, Venezia, 2013.

2 "Venezia e la sua Laguna” è un binomio inseparabile, tanto da diventare l'oggetto stesso della nomina all'interno della lista dei siti patrimonio dell'Umanità (UNESCO, iscrizione del 1987, Id n. 394 1987, cfr. http:/ /whc.unesco.org/en/list/394). Tra gli altri vale la pena ricordare il Lane (F. LANE, Venice, A Maritime Republic, Baltimore, 1973, p. 1) per il quale fin dall'inizio del suo lavoro propone l'idea che l'isolamento nelle lagune sia la ragione dei valori democratici e libertari della città: "The distinctiveness of the environment in which the Venetians built gave an obviously unique quality to their city's charm. Its watery setting contributed also to an aristocratic tradition of liberty".

${ }^{3}$ Le origini di Venezia come prodotto delle fughe in laguna sono bene descritte in Wikipedia sia nella versione italiana (http://it.wikipedia.org/wiki/ Venezia) che nelle principali lingue straniere: "historians .. agree that the original population of Venice consisted of refugees from Roman cities near Venice and from the undefended countryside, who were fleeing successive waves of Germanic and Hun invasions" (http://en.wikipedia.org/wiki/Venezia); “..Elle fut donc initialement un refuge de la civilisation romano-byzantine mais au fur et à mesure de son développement, son autonomie s'accrut pour aboutir à l'indépendance". (http://fr.wikipedia.org/wiki/Venezia); interessante notare come nella versione tedesca vi sia solo un accenno veloce alle grandi migrazioni germaniche (Völkerwanderung Flüchtlinge) e invece si descriva l'origine della città come il prodotto di un avamposto Bizantino "Byzantinischer Außenposte" (http://de.wikipedia.org/wiki/Venezia).

${ }^{4}$ Numerosi sono gli studi a cui si può fare riferimento. Si ricordano qui, per completezza, le sintesi di Ortalli: G. ORTALLI, Nascere sull'acqua. La lunga genesi di Venezia, in L'acqua nei secoli altomedievali : Spoleto, 12-17 aprile 2007, Atti della LV Settimana di Studio, Centro di Studi sull'Alto Medioevo di Spoleto, 2008, Spoleto, pp. 141-182; tra gli altri, si veda il contributo più recente di A. CARILE, Il ducato venetico fra ecumene bizantina e società locale, in La Venetia tra antichità e alto medievo, Roma, 1988, pp. 89-109; di carattere divulgativo sono i lavori di G. ORTALLI, G. SCARABELLO, Breve storia di Venezia, Pisa, 1990 e di S. GASPARRI, Come nasce Venezia, in Storia del veneto. 1. Dalle origini al Seicento, a cura di C. FUMIAN, A. VENTURA, Bari, 2000, pp. 71-86.

${ }^{5}$ La laguna descritta da Giovanni Diacono è una laguna popolata, ricca, dove "abitare" appare naturale come in qualsiasi altra "civitas", cfr. GIOVANNI DIACONO, Istoria Veneticorum, ed. e trad. a cura di A. BERTO, Bologna, 1999.

${ }^{6}$ Cassiodoro, nella celebre lettera ai Venetici del 537, sottolinea gli aspetti di integrazione tra ambiente naturale e soluzioni tecnico -scientifiche per gli abitati e i trasporti lagunari, VARIE, XII, 24 = Magni Aurelii Cassiodori Senatoris, Variorum libri duodecim, in "Monumenta Germaniae Historica", Auctorum antiquissimorum tomus XII, recensuit T. Mommsen, Berolini 1894 (editio nova 1961).

${ }^{7}$ Fondamentale in tal senso è stato il contributo di Roberto Cessi. Tra gli altri titoli si ricorda qui R. CESSI, Le origini del ducato veneziano, Napoli, 1951. ${ }^{8}$ Cfr. W. DORIGO, La Cultura Carolingia nella prima “Capella Sancti Marci”, in Hortus Artium Medievalium, vol. 8, 2002, pp. 149-157; D. CALAON, L'intreccio della nascente Venezia. Sculture e marmi dei primi Dogi conservati presso i Musei di Piazza San Marco, in Dalla catalogazione alla promozione dei beni archeologici.I progetti europei come occasione di valorizzazione del patrimonio culturale veneto, Venezia, 2014, pp. 233-244.

${ }^{9}$ Cfr. S. GELICHI, Venezia tra archeologia e storia: la costruzione di una identita urbana, in Le citta italiane tra la tarda Antichita e l'alto Medioevo, Atti del Convegno, Ravenna, 26 - 28 febbraio 2004, a cura di A. Augenti, Firenze, 2006, pp. 151 - 183; M. STAHL, Zecca. The Mint of Venice in the Middle Ages, Baltimore and London, 2000.

${ }^{10} \mathrm{Cfr}$. G. RAVEGNANI, I Bizantini in Italia, Bologna 2004.

${ }^{11}$ Cfr. per Cittanova: D. CALAON, Cittanova (VE): Analisi GIS, IV Congresso Nazionale di Archeologia Medievale. Scriptorium dell'Abbazia, Abbazia di San Galgano (Chiusdino, Siena), 26-30 Settembre 2006, a cura di R. Francovich, M. Valenti, Firenze, pp. 216-224; D. CALAON, Cittanova, Flourishing Places in North-Eastern Italy. Towns and emporia between Late Antiquity and Carolingian Age, S. GELICHI, in Post-Roman Towns and Trade in Europe, Byzantium and the Near East. Vol. 1. The Heirs of Roman West, edited by J. HENNING, pp. 77-104, Berlin - New York, 2007, pp. 88-93; Per il problema del nome: G. ROSADA, Da Civitas Nova a Heracliana. Il possible caso di propaganda alle origini storiche di Venezia, “Aquileia Nostra”, LVII, 1986, pp. 910-928. ${ }^{12}$ Tra le centinaia di viaggi e spostamenti di genti e merci documentati da Micheal McCormick per il Mediterraneo tra VIII e IX secolo, appare evidente come la presenza di navi veneziane ad Alessandria d'Egitto costituisca un elemento di normalità, che segnala un rapporto commerciale più che consolidato proprio con il mondo arabo: M. McCORMICK, Origins of the European Economy, Communication and Commerce, AD 300-900, Cambridge, 2001, pp. 268-245 e 523-529.

${ }^{13}$ L LECIEJEWICZ (a cura di), Torcello. Nuove ricerche archeologiche, Roma, 2000; Ma anche il seguente: L. LECIEJEWICZ, E. TABACZYSKA, S. TABACZYSKY (a cura di), Torcello. Scavi 1961-62, Roma, 1977.

${ }^{14}$ Si veda l'ultimo e aggiornato contributo di: G. ROSADA, M. ZABEO, ...Stagna...inrigua aestibus maritimis...Sulla laguna di Venezia ovvero su un comprensorio a morfologia variabile, in Histria Antiqua, 21, 2012, pp. 241-262.

${ }^{15}$ D. CALAON, La Venetia marittima tra il VI e il IX sec.: mito, continuità e rottura, in Dalla catalogazione alla promozione dei beni archeologici.I progetti europei come occasione di valorizzazione del patrimonio culturale veneto, Venezia, 2014, pp. 53-61; D. CALAON, G. BISCONTIN, E. ZENDRI E., M. SGOBBI, 2013, Torcello 2012. Valorizzare, preservare e conoscere mentre si scava. Il Punto di vista degli archeologi, in "Conservazione e Valorizzazione dei siti Archeologici. Approcci Scientifici e problemi di metodo", atti del Convegno di Studi, Bressanone 9-12 luglio 2013, Venezia, pp. 155-209.

${ }^{16}$ D. CALAON, Quando Torcello era abitata, Venezia 2013.; D. CALAON, Età tardo-antica e alto medioevo: magazzini, èlites e insediamento, in Torcello scavata, patrimonio condiviso. Lo scavo 2012-2013, a cura di D. Calaon, E. Zendri, G. Biscontin, Venezia, 2014.

${ }^{17}$ Cfr. D. CALAON a pp. 395-398 di S. GELICHI S., D. CALAON, Comacchio: la storia di un emporio sul delta del Po, in "Genti del Delta da Spina a Comacchio. Catalogo della Mostra,Comacchio 2006-2007”, a cura di Gelichi S., Berti F., Ortalli J., Ferrara, 2007, pp. 387-416.

${ }^{18} \mathrm{Si}$ rimanda alle pagine di M. McCORMICK, op. cit, pp. 758 e 761-768, dove si fa forza con chiarezza l'ipotesi che una delle merci di più grande valore alla base delle relazioni commerciali tra mondo Carolingio e mondo Arabo sia costituita da schiavi.

${ }^{19}$ D. CALAON, Prima di Venezia. Terre, acque e insediamenti. Strumenti GIS per la comprensione delle trasformazioni territoriali tra tarda antichità e altomedioevo. Tesi di Dottorato in "Archeologia e Storia dei paesi del mediterraneo", XVIII ciclo, AA 2002/3 - 2004/5, tesi di dottorato, relatore Gelichi S., "Dipartimento di Scienze dell'Antichità e del Vicino Oriente", Ca' Foscari, Venezia, 2006.

${ }^{20}$ S. GELICHI, D. CALAON, E. GRANDI, C. NEGRELLI, “... castrum igne combussit...”. Comacchio tra la Tarda Antichità el'Alto Medioevo, "Archeologia Medievale", XXXIII, 2006, pp- 19-48; GELICHI S. (a cura di), L'Isola del vescovo. Gli scavi archeologici intorno alla cattedrale di Comacchio, Firenze, 2009. 
${ }^{21}$ G. P. BROGIOLO, A. CAGNANA, Le fortificazioni del castrum di Grado, in Ai confni dell'Impero. Insediamenti e fortificazioni bizantine nel Mediterraneo Occidentale (VI - VIII secolo), Bordighera, 14-17 marzo, 2002, Bordighera, 2002, pp. 467-508.

${ }^{22}$ S. GELICHI, C. MOINE, Peregrinazioni in sconfinati deserti: Quale archeologia per i monasteri della laguna Veneziana?, in Hortus Artium Medievalium, vol. 19, 2013, pp. 133-154; D. CALAON, M.FERRI, Il monastero dei Dogi. SS. Ilario e Benedetto ai margini della Laguna veneziana, in Missioni Archeologiche e Progetti di Ricerca e Scavo dell'Università Ca' Foscari - Venezia, VI Giornata di Studio, Venezia 12 maggio 2008, Dipartimento di Scienze dell'Antichità e del Vicino Oriente, a cura di S. Gelichi, Venezia, 2008,pp. 185-197; D. CALAON, M. FERRI, C. BAGATO, SS. Ilario e Benedetto (IX secolo). Un monastero del nascente dogado veneziano tra terra e laguna, in V congresso nazionale di archeologia medievale: Palazzo della Dogana, Salone de Tribunale (Foggia), Palazzo dei Celestini, Auditorium (Manfredonia), 30 settembre - 3 ottobre 2009, a cura di G. Volpe, G. Favia, Firenze, 2009, $498-504$. ${ }^{23}$ Per una sintesi e nuovi important dati sul paesaggio costiero tra Po e Livenza, si consideri C. NEGRELLI, P. MOZZI (a cura di), Paesaggi antichi e potenziale archeologico, in “Archeologia e paesaggio nell'area costiera veneta: conoscenza, partecipazione e valorizzazione", Regione del Veneto, 2013, pp. 18-85. Il volume, frutto di recentissime indagini di superficie e ricco di analisi geomorfologiche, riconsidera in un'ottica di continuità (sia cronologica, che territoriale tra entroterra e laguna) siti chiave nell'insediamento costiero, a partire da S. Basilio, presso Ariano Polesine, fino a Jesolo.

${ }^{24}$ Si veda il recente P. MOZZI, S. PRIMON, Torcello e la morfologia della laguna tra l'età romana e il medioevo, in Torcello scavata, patrimonio condiviso. Lo scavo 2012-2013, a cura di D. Calaon, E. Zendri, G. Biscontin, Venezia, 2014.

${ }^{25}$ Confronta E. MIGLIARO, A proposito di CTH IX 30. Alcune riflessioni sul paesaggio tardo antico, "Archeologia Medievale”, XXII, 1995, pp. $475-485$.

${ }^{26}$ S. GELICHI S., D. CALAON, 2007,op. cit.

${ }^{27}$ D. CALAON, Cittanova, op. cit.;M. DE FRANCESCHINI, Le ville romane della 10. Regio (Venetia et Histria): catalogo e carta archeologica dell'insediamento romano nel territorio, dall'età repubblicana al tardo impero, Roma 1998.

${ }^{28}$ Si vedano le pagine di S. GASPARRI, Anno 713. La leggenda di paulicio e le origini di Venezia, in Venezia: I giorni della Storia, a cura di U. Israel, Roma, 2001, pp. 27-46.

${ }^{29}$ F. SARTORI, Antoninus tribunus in un'epigrafe inedita di Iesolo (Venezia), in Adriatica praehistorica et antiqua, in "Miscellanea G. Novak dicata", Zagreb 1970, p. 587-589.

${ }^{30}$ L. CALVELLI, Le iscrizioni di epoca romana reimpiegate nel complesso episcopale di Torcello, in Omaggio a Torcello. La storia, l'archeologia e l'arte nel millenario della basilica di Santa Maria Assunta, a cura di G. Caputo, A. Enzo, Venezia, 2009; L. CALVELLI, Il reimpiego epigrafico a Venezia: $i$ materiali provenienti dal campanile di San Marco., in "Antichità Altoadriatiche", 2009, vol. 74, pp. 179-202.

${ }^{31}$ Il Congresso From one sea to another: Trading places in the European and mediterranean Early Middle Ages, Comacchio 2009, è stato organizzato da Sauro Gelichi, direttore scientifico degli scavi di Comacchio e Richard Hodges, teorico degli empori del nord Europa: i lavori hanno voluto intenzionalmente percorrere le similitudini (e le differenze) tra nord e sud nell'Europa pre e post-carolingia. La Venetia, dunque, è stata considerata come una delle regioni europee e mediterranee che concorrono nella ricomposizione di una nuova economia caratteristica del medioevo, superando una forte connotazione di "bizantinità" e di "classicità" che aveva tradizionalmente condizionato i lavori archeologici e storici condotti nella regione, cfr. S. GELICHI, R. HODGES (eds.), From one sea to another: Trading places in the European and mediterranean Early Middle Ages. Proceedings of the International Conference, Comacchio 27th-29th Marzo 2009, Turnhout, 2013.

${ }^{32}$ Il termine processo genetico è felicemente usato in G. ORTALLI, Torcello e la genesi di Venezia, in "Torcello. Alle origini di Venezia tra Occidente e Orienete", a cura di G. Caputo, G. Gentili, Venezia, 2009.

${ }^{33}$ L. LECIEJEWICZ, op. cit, L. LECIEJEWICZ, E. TABACZYSKA, S. TABACZYSKY, op. cit.

${ }^{34}$ Per le fasi antiche di Torcello e per la cronologia di Santa Maria Assunta, fondamentali gli interventi di M. DE MIN, Edilizia altomedioevale e medioevale nel territorio lagunare. Nuovi dati conoscitivi dai cantieri di restauro, in Tra due elementi sospesa. Venezia, costruzione di un paesaggio urbano, Venezia, 2000, pp. 98-133;, M. DE MIN Nuovi dati sullo sviluppo insediativo lagunare nel periodo delle origini della Civitas Veneciarum. Forme e tecniche del costruire, in Quaderni di Archeologia del Veneto, serie speciale II, 2006, pp. 227-243.

${ }^{35}$ Ad esempio in M. DE MIN, Edilizia altomedioevale, op. cit., Ma è questa un opinione comune che emerge in buona parte delle relazioni di scavo.

${ }^{36}$ Così ad esempio nelle attente pagine di G. ORTALLI, Torcello e la genesi di Venezia, op. cit., p. 26 e p.29; la stessa linea interpretativa pervade la recente (2008) documentata analisi sulla genesi di Venezia dello stesso autore, cfr. ID., Nascere sull'acqua. La laguna e la genesi di Venezia, in “L'acqua nei secoli altomedievali", Spoleto: Centro Italiano di Studi sull'Alto Medioevo, Spoleto, 2008, pp. 141-182.

${ }^{37}$ E' pur vero che secondo E. CROUZET-PAVAN, La mort lente de Torcello: Histoire d'une cité disparue, Paris, 1995, questo scarto corrisponderebbe al frutto di una strategia politica precisa, volta a oscurare parte della storia delle origini della città. Nelle cancellerie veneziane medievali vi sarebbe stata la precisa volontà di oscurare Torcello per esaltare la centralità mitica di Rialto/Venezia.

${ }^{38}$ D. CALAON, Altino (VE). Strumenti diagnostici (GIS e DTM) per l'analisi delle fasi tardoantiche ed altomedievali, in "Le Missioni archeologiche di Ca'Foscari, V giornata di studio. 2006", a cura di A.P. Zaccaria Ruggiu, Venezia, 2006, pp. 143-158.

${ }^{39}$ M. MARITAN, Le analisi archeobotaniche presso Torcello e la laguna centrale veneziana: lo stato dell'arte, in Torcello scavata, patrimonio condiviso. Lo scavo 2012-2013, a cura di D. Calaon, E. Zendri, G. Biscontin, Venezia, 2014.

${ }^{40}$ S. GELICHI, Infrastrutture marittime nell'alto medioevo: una prospettiva archeologica, in "Spoleto, Centro Italiano di studi sull'altomedioevo, vol. LV, 2008, pp. 283-317.

${ }^{41}$ M. D’AGOSTINO, S. MEDAS 2005 La navigazione nella Laguna di Venezia in epoca romana: nuove evidenze dall'archeologia subacquea, in “Journal of Ancient Topography", XV, pp. 37 - 54.

${ }^{42}$ M. D'AGOSTINO ET ALII 2008 Il paesaggio costiero antico nella Laguna nord di Venezia: recenti acquisizioni dall'archeologia subacquea, in Terre di mare. L'archeologia dei paesaggi costieri e le variazioni climatiche, Atti del Convegno internazionale di Studi, Trieste, 8 - 10 novembre 2007, a cura di R. Auriemma e S. Karinja, Udine pp. 340 - 348.

${ }^{43}$ Come ad esempio a Corte Cavanella, L. SANESI MASTROCINQUE, L'insediamento romano di corte Cavanella (Loreo), in Misurare la terra: centuriazione e coloni nel mondo romano. Il caso Veneto, Roma, 1984, pp. 109-116.

${ }^{44}$ D. CALAON, Altino .., op. cit..

${ }^{45}$ Cfr. NINFO ET ALII 2009 The map of Altinum, ancestor of Venice, in “Science”, Brevia, CCCXXV, p. 577 , P. MOZZI, A. FONTANA, A. NINFO, F. FERRARESE, in Altino dal cielo. La città telerivelata, a cura di M. Tirelli, G. Cresci Marrone, Roma, 2011.

${ }^{46}$ Per il porto di Ravenna, M. LILLI, Le attrezzature portuali di Ravenna durante l'età romana e bizantina. Progettualità e trasformazione del paesaggio, in "Città e monumenti nell'Italia antica", a cura di S. Quilici Gigli, Roma 1999, pp. 17-42; E. CIRELLI, Ravenna: archeologia di una città, Firenze 2008; ID. Roma sul mare e il porto Augusteo di Classe, in "Ravenna e l'Adriatico dalle origini all'età romana" a cura F. Boschi, 2013, pp. 109-121.

${ }^{47}$ In futuro con serie analisi paleo demografiche su cimiteri tardo antichi e altomedievali sarà forse possibile rispondere a tale domanda. A Torcello è stato scavato un importante cimitero basso medievale e alcune tombe altomedievali, (L. LECIEJEWICZ, E. TABACZYSKA, S. TABACZYSKY, op. cit.), 
ma manca ancora uno studio sistematico delle caratteristiche demografiche antiche. Manca uno scavo di un cimitero tardo antico e altomedievale, che permetta l'acquisizione sistematica di tutti i dati necessari per la determinazione della demografia antica, non rinunciando a campagne di studio sul DNA antico e con analisi di geochima isotopica, oggi molto sviluppate e capaci di rispondere positivamente alle domande sulla mobilita e sulla composizione (anche etnica) dei gruppi umani.

${ }^{48}$ L. LECIEJEWICZ, E. TABACZYNSKA, S. TABACZYNSKI, op. cit, pp. 80-84.

${ }^{49}$ DE MIN M. Edilizia .. , op. cit.; M. DE MIN, Nuovi dati ..., op. cit.; M. DE MIN, Venezia e il territorio lagunare, in Ritrovare restaurando. Rinvenimenti e scoperte a Venezia e in laguna, Cornuda, 2000, pp. 15-25.

${ }^{50}$ G. ROSADA, M. ZABEO, op. cit.; M. D'AGOSTINO, A. TONIOLO, Una struttura arginale di epoca romana dinanzi l'isola di Burano (Venezia), in “Archeologia delle Acque”, V, pp. 121 - 134; L. FOZZATI, A. TONIOLO A. 1998 Argini-strade nella Laguna di Venezia, in Bonifiche e drenaggi, pp. 197 - 208.

${ }^{51}$ Cfr. MART., IV, 25, 1-2.

${ }^{52}$ E. GRANDI, Ceramiche fini da mensa dalla laguna veneziana. I contesti di San Francesco del Deserto e Torcello, in La circolazione delle ceramiche nell'Adriatico tra Tarda Antichità e Altomedioevo (III Incontro di Studio CER.AM.IS), a cura di S. Gelichi, C. Negrelli, Mantova, 2007, pp. 127-153.

${ }^{53}$ C. MALAGUTI, Il materiale ceramico, in Torcello scavata, patrimonio condiviso. Lo scavo 2012-2013, a cura di D. Calaon, E. Zendri, G. Biscontin, Venezia, 2014.

${ }^{54}$ M. FERRI, Reperti vitrei altomedievali dagli scavi di Torcello e San Francesco del Deserto - Venezia, in "Journal of Glass Studies”, vol. 48; p. 175-191.

${ }^{55}$ In laguna, a San Francesco del Deserto ci sono materiali anforici e da mensa a partire dal IV sec. d.C. (M. DE MIN, Edilizia altomedioevale, op. cit). Anche a San Lorenzo d'Ammiana la struttura più antica, probabilmente collegabile ad una villa rustica lagunare, pare non avere fasi prima del V secolo, Cfr. S. GELICHI, C. MOINE (a cura di), Isole fortunate? La storia della laguna nord di Venezia attraverso lo scavo di San Lorenzo di Ammiana, in "Archeologia medievale", XXXIX, 2012, pp. 9 -56.

${ }^{56}$ A. J. AMMERMAN, C. E. MCCLENNEN, Venice before San Marco. Recent studies on the Origins of City., New York 2001; A. J. AMMERMAN, C. E. MCCLENNEN, M. DE MIN, Housley R. 1999, Sea-level change and the archaeology of early Venice, in “Antiquity”, LXXIII, pp. 303 - 312.

${ }^{57}$ A. J. AMMERMAN, C. E. MCCLENNEN, Venice.., op. cit., p. 17.

${ }^{58}$ A. MARACANTE, Il vetro, in Torcello scavata, patrimonio condiviso. Lo scavo 2012-2013, a cura di D. Calaon, E. Zendri, G. Biscontin, Venezia, 2014.

${ }^{59}$ L. LECIEJEWICZ, E. TABACZYNSKA, S. TABACZYNSKI, Op. cit., pp. 114-131, 175-177; M. MININI, M. VERITÀ, S. ZECCHIN, Materiali vitrei del IV-XV secolo nel territorio della Laguna di Venezia: indagini archeologiche ed archeometriche, Rivista della Stazione sperimentale del vetro 5, 2008 , pp. 15-32.

${ }^{60}$ K. SEETAH, A. PLUSKOWSKI, Resti archeozoologici, in Torcello scavata, patrimonio condiviso. Lo scavo 2012-2013, a cura di D. Calaon, E. Zendri, G. Biscontin, Venezia, 2014.

${ }^{61}$ C. WICKHAM, Framing the Early Middle Ages. Europe and the Mediterranean, 400-800, Oxford 2005.

${ }^{62}$ S. GELICHI, D. CALAON D., E. GRANDI, C. NEGRELLI 2012, History of a forgotten Town: Comacchio and its Archaeology, in Gelichi. S, Hodges. R. (eds).

"From One Sea to the Other: trading Places in the European and Mediterranean Early Middle Ages" Brepols, Turnhout, pp. 169-206.

\section{EKOLOGIJA VENETIE PRIJE VENECIJE: LJUDI, VODA I ARHEOLOGIJA}

\section{SAŽETAK}

Odnos između Venecije i mora je očigledan i u svim aspektima jako povezan s poviješću Serenissime. Nova i starija arheološka istraživanja često su naglašavala kako je topografski smještaj unutar lagune s izlazom na Jadran, značio za grad mnogo više od jednostavnog i uspješnog sjedišta. Voda i more vitalni su elementi gospodarstva grada: u ekološkoj studiji ovog važnog odnosa grada prvo s lagunarnim prostorom a zatim i morskim, ovdje se istražuju elementi koji određuju, tijekom dužeg vremena, izvanredni razvoj jedinstvenog političkog i ekonomskog organizma.

Koristeći širu lepezu podataka, i kontekstualizirajući grad kasne antike i ranog srednjeg vijeka unutar područja Venetia maritima, moguće je sakupiti cijeli niz tipoloških i tehnoloških detalja s lokaliteta koji sudjeluju u formaciji složenog organizma koji će biti određen kao Venecija, polazeći od 9.-10.stoljeća. Comacchio, Cittanova, Torcello, ali i Grado, odnosno Aquileia, Classe odnosno Ravenna, omogućuju nam čitanje "venecijanske" stratigrafije (one koja pripada gradu, ali iznad svega one laguni) u svjetlu niza mehanizma gdje tehnološki aspekti, prijenos znanja u konstrukciji, definiranje zajednice lagune u odnosu na okolinu prolaze strelovite promjene, a koja nije vezana za čestu i pojednostavljenu "sklonost Bizantu".

U čitanju topografske organizacije i izbora lokacija za izvorišna mjesta izgradnje arheološkim metodama ključne su teme: lokalno znanje naslijeđeno od kasne antike, tehničke vještine gradnje brodova (od najjednostavnijih do najsloženijih, u dobu u kojem su trgovačke flote razrijeđene u cijelom Mediteranu), politički izbori osnivanja prebivališta $\mathrm{u}$ prostru lagune.

Venecija prije Venecije, koju arheologija dobro restituira, više sliči selu sjeverne Europe izgrađenom iz drva, nego gradu iz kamena bizantskog istoka. Ona ima mnogo više kontakata i sličnosti sa zaleđem rijeke Po nego s mitskim Istokom. $\mathrm{U}$ isto vrijeme, materijalna kultura i kvaliteta robe $\mathrm{u}$ opticaju približava Veneciju Mediteranu u naglome usponu, gdje je uloga političkih, vojnih i crkvenih elita u stvaranju temelja novog gospodarstva na europskoj razini velika.

Prevela: Tea Gorup 